ARTICLE

Received 5 Aug 2015 | Accepted 31 Mar 2016 | Published 6 May 2016

DOI: $10.1038 /$ ncomms11483

OPEN

\title{
Deregulation of mitochondrial F1FO-ATP synthase via OSCP in Alzheimer's disease
}

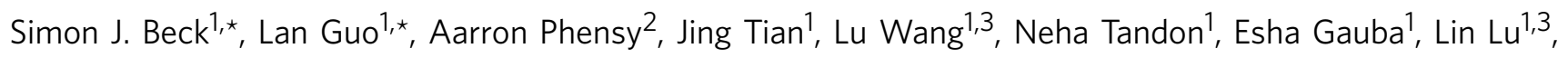
Juan M. Pascual ${ }^{4}$, Sven Kroener ${ }^{2} \&$ Heng Du, ${ }^{1,3}$

F1FO-ATP synthase is critical for mitochondrial functions. The deregulation of this enzyme results in dampened mitochondrial oxidative phosphorylation (OXPHOS) and activated mitochondrial permeability transition (mPT), defects which accompany Alzheimer's disease (AD). However, the molecular mechanisms that connect F1FO-ATP synthase dysfunction and $A D$ remain unclear. Here, we observe selective loss of the oligomycin sensitivity conferring protein (OSCP) subunit of the F1FO-ATP synthase and the physical interaction of OSCP with amyloid beta $(A \beta)$ in the brains of $A D$ individuals and in an $A D$ mouse model. Changes in OSCP levels are more pronounced in neuronal mitochondria. OSCP loss and its interplay with $A \beta$ disrupt F1FO-ATP synthase, leading to reduced ATP production, elevated oxidative stress and activated mPT. The restoration of OSCP ameliorates A $\beta$-mediated mouse and human neuronal mitochondrial impairments and the resultant synaptic injury. Therefore, mitochondrial F1FO-ATP synthase dysfunction associated with AD progression could potentially be prevented by OSCP stabilization.

\footnotetext{
${ }^{1}$ Department of Biological Sciences, The University of Texas at Dallas, 800W. Campbell Road, Richardson, Texas 75080, USA. ${ }^{2}$ School of Behavioral and Brain Sciences, The University of Texas at Dallas, 800W. Campbell Road, Richardson, Texas 75080, USA. ${ }^{3}$ Department of Neurology, Shandong Provincial Hospital Affiliated to Shandong University, 324 Jingwu Weiqi Road, Jinan, Shandong 250021, China. ${ }^{4}$ Department of Neurology and Neurotherapeutics, The University of Texas Southwestern Medical Center, 5323 Harry Hines Boulevard, Richardson, Texas 75390, USA. * These authors contributed equally to this work. Correspondence and requests for materials should be addressed to L.G. (email: lan.guo@utdallas.edu) or to H.D. (email: heng.du@utdallas.edu).
} 
C linical observations have suggested that mitochondrial dysfunction is among the earliest manifestations of Alzheimer's disease (AD) and constitutes a hallmark pathological feature of this neurological disorder ${ }^{1,2}$. Previous studies have also identified impaired mitochondrial oxidative phosphorylation (OXPHOS) as a feature of mitochondrial defects in $\mathrm{AD}$ individuals $\mathrm{s}^{2,3}$ and $\mathrm{AD}$ animal models ${ }^{4-6}$. Compromised mitochondrial OXPHOS efficiency results in lowered mitochondrial bioenergetics and exaggerated production of free radicals ${ }^{7}$. Indeed, ATP deficiency and oxidative damage are characteristics of brains from $\mathrm{AD}$ patients ${ }^{2}$. Impaired mitochondrial OXPHOS efficiency is closely associated with dysfunction of mitochondrial respiratory enzymes, including mitochondrial complex I to IV, as well as the defect of F1FO-ATP synthase. Previous studies have mostly focused on the dysfunction of mitochondrial complex IV in $\mathrm{AD}^{8-10}$. However, this concept has been recently challenged ${ }^{11-13}$. In fact, in addition to mitochondrial respiratory enzyme defects, increasing evidence implicates the dysfunction of mitochondrial F1FO-ATP synthase in $\mathrm{AD}^{14-17}$

The mitochondrial F1FO-ATP synthase, which includes three components (F1, FO and the peripheral stalk), is a critical mitochondrial OXPHOS enzyme involved in the regulation of mitochondrial ATP production and in the maintenance of the mitochondrial membrane potential ${ }^{18,19}$. The F1FO-ATP synthase can both synthesize ATP and degrade ATP when operating in reverse to generate proton backflow, increasing mitochondrial membrane potential when it is critically low ${ }^{19-2}$. In addition to its vital function in mitochondrial OXPHOS, recent studies have shown that this enzyme contributes to the formation of the mitochondrial permeability transition pore $(\mathrm{mPTP})^{22,23}$ through the interaction of its oligomycin sensitivity conferring protein (OSCP) subunit with cyclophilin D (CypD), the key regulator of $\mathrm{mPTP}^{22-24}$. Extensive formation of $\mathrm{mPTP}$ is a severe mitochondrial pathological event that leads to collapsed mitochondrial membrane potential $(\mathrm{m} \Delta \Psi)$, decreased mitochondrial OXPHOS capacity, elevated reactive oxygen species (ROS) generation and, eventually, cell death ${ }^{25}$. Indeed, mPTP activation is thought to be a key mechanism of mitochondrial stress in $\mathrm{AD}$ and has been proposed to underlie its characteristic synaptic dysfunction and cognitive decline $e^{5,26}$. Given its role in mitochondrial OXPHOS ${ }^{18}$ and $\mathrm{MPTP}$ formation $^{21-23}$, the deregulation of mitochondrial F1FO-ATP synthase may predispose to compromised OXPHOS efficiency and sensitized mPTP formation, which are two hallmark mitochondrial defects in AD. However, to date information on the dysfunction of F1FO-ATP synthase in $\mathrm{AD}$ has remained limited. Accordingly, the underlying molecular mechanisms causing the defect of this enzyme in AD remain unresolved.

In this study, we compare the levels of major F1FO-ATP synthase subunits in the brains from $\mathrm{AD}$ individuals, mild cognitive impairment (MCI) patients and non-AD control subjects, and find a selective decrease in the levels of OSCP during the progression of $\mathrm{AD}$. We also find that, in a mouse model of AD that overexpresses the human form of amyloid beta $(\mathrm{A} \beta)$, the loss of OSCP is more prominent in synaptic mitochondria. In addition to OSCP loss, we also detect a direct physical interaction between OSCP and $A \beta$ in the brains from $\mathrm{AD}$ cases, as well as in AD mice. Such OSCP aberrations disrupt F1FO-ATP synthase stability, leading to severe mitochondrial dysfunction and synaptic injury. Further in vivo studies show the deleterious impact of F1FO-ATP synthase dysfunction on the development of mitochondrial defects in $\mathrm{AD}$ mice. Importantly, the restoration of OSCP ameliorates the A $\beta$-mediated mitochondrial dysfunction and synaptic injury in mouse or human neurons, further supporting the role of OSCP deregulation in mitochondrial dysfunction under $\mathrm{A} \beta$-rich conditions. Therefore, mitochondrial F1FO-ATP synthase dysfunction that results from OSCP aberrations may constitute a primary AD event that can be prevented by OSCP protection, suggesting OSCP as a potential new therapeutic target for $\mathrm{AD}$.

\section{Results}

Loss of OSCP in AD subjects and 5xFAD mice. To examine changes of F1FO-ATP synthase in AD brains, we used immunoblotting to compare the expression levels of the major subunits of the mitochondrial F1FO-ATP synthase in protein extracts from the temporal lobe of non-AD, MCI, and $\mathrm{AD}$ subjects (Supplementary Table 1). Among the F1FO-ATP synthase subunits tested here, OSCP was slightly reduced in MCI brains, while this decrease was exaggerated in $\mathrm{AD}$ patients (Fig. 1a). Notably, the expression levels of the other major F1FO-ATP synthase subunits, including $a, b, c, \alpha$, and $\beta$ were not significantly changed in either MCI or AD brains (Supplementary Fig. 1a,b), implying that the OSCP loss is not likely a result of F1FO-ATP synthase reduction in $\mathrm{AD}$. Next, we performed histological studies on brain sections from the temporal lobes of $\mathrm{AD}$ and control subjects. Our results showed a dramatic reduction of OSCP expression in neurons from the temporal lobe of $\mathrm{AD}$ individuals (Fig. 1b). A similar reduction of OSCP also occurred in an AD mouse model (5xFAD mice). To determine whether synaptic mitochondria are more vulnerable to OSCP loss under conditions of elevated $A \beta$, we separated synaptic mitochondria and nonsynaptic mitochondria from $5 x F A D$ mice at 4 and 9 months old that mimic the amyloidopathy and behavioral changes typical of the early-, and middle to late-stages of $\mathrm{AD}$, respectively ${ }^{27}$. The purity of isolated mitochondria was verified by means of the detection of high abundance of the mitochondrial protein voltage-dependent anion channel (VDAC) without contamination of $\beta$-actin or synaptic vesicles (Supplementary Fig. 2). Synaptic mitochondria demonstrated a significant OSCP decrease in young $5 \mathrm{xFAD}$ mice, and this OSCP loss was exacerbated with age (Fig. 1c). However, a significant OSCP reduction in nonsynaptic mitochondria was detected only in old $5 x F A D$ mice (Fig. 1d), suggesting that in A $\beta$-rich environments synaptic mitochondria are more susceptible to OSCP loss. In sharp contrast, the expression levels of $\mathrm{a}, \mathrm{b}$ and $\mathrm{c}$, as well as $\alpha$ and $\beta$ subunits remained unaltered in either synaptic or nonsynaptic mitochondria from $5 x F A D$ mice at any tested age (Supplementary Fig. 3a-d), indicating that OSCP expression is selectively suppressed in parallel with the manifestation of AD-like symptoms in $5 x F A D$ mice.

OSCP loss impacts mitochondrial and synaptic function. To investigate the impact of OSCP loss on neuronal mitochondrial function we genetically downregulated OSCP expression in primary cultured mouse neurons by using specific OSCP shRNA. Nontarget shRNA was used as control. Quantitative analysis showed substantially decreased OSCP levels in OSCP knockdown neurons (OSCP KD, Fig. 2a). The key functions of mitochondrial F1FO-ATP synthase are to maintain $\mathrm{m} \Delta \Psi$ and to produce ATP. To determine the impact of OSCP loss on F1FO-ATP synthase function, we first measured $\mathrm{m} \Delta \Psi$ in OSCP knockdown neurons via staining with tetramethylrhodamine methyl ester (TMRM), an indicator of $\mathrm{m} \Delta \Psi$ (ref. 5). In comparison with control neurons, OSCP knockdown neurons exhibited significantly decreased intensities of TMRM (Fig. 2b), suggesting a collapsed $\mathrm{m} \Delta \Psi$ in response to OSCP loss. Furthermore, in agreement with reduced $m \Delta \Psi$, ATP generation was markedly reduced in OSCP knockdown neurons (Fig. 2c), suggesting deregulation of mitochondrial OXPHOS efficiency. In view of the close 
correlation between compromised OXPHOS efficiency and mitochondrial ROS production, we next compared mitochondrial ROS levels by staining with MitoSox Red, a specific fluorescent indicator of mitochondrial superoxide abundance ${ }^{5,6}$. Our results showed significantly elevated MitoSox Red intensity in mitochondria from OSCP knockdown neurons (Fig. 2d). In addition, in comparison to the control neurons OSCP downregulated neurons also displayed significantly reduced mitochondrial population in neurites (Fig. 2e).

OSCP deregulation has also been previously linked to $\mathrm{mPTP}$ formation ${ }^{22-24}$. To determine whether the loss of OSCP sensitizes mPTP opening, we subjected neurons to calcein AM-cobalt chloride quenching assay ${ }^{28}$. When we exposed the neurons to ionophore, which increases mitochondrial calcium overloading to trigger mPTP formation ${ }^{29}$, OSCP knockdown neurons exhibited larger reductions in mitochondrial calcein intensity than control neurons (Fig. 2f). CypD is a known regulator of $\mathrm{mPTP}^{25}$. Inhibiting CypD pharmacologically with cyclosporin A (CsA) substantially suppressed mPTP formation in control neurons; however, mPTP activation associated with OSCP downregulation was not blocked by the addition of CsA (Fig. 2f). These results seem to implicate that mPTP activation induced by loss of OSCP does not rely on the functional status of CypD. This was further supported by unaltered CypD expression levels in OSCP knockdown neurons detected by immunoblotting in parallel with calcein AM-cobalt chloride quenching assay (Supplementary Fig. 4a). Furthermore, by downregulating
OSCP in CypD-deficient neurons, we found that mPTP activation resulting from OSCP deficiency was not protected by depletion of CypD (Supplementary Fig. 4b,c), which is in agreement with the abolished effect of CsA treatment (Fig. 2f). Taken together, these data suggest that the loss of OSCP sensitizes mPTP opening regardless of the functional status of CypD.

Because the major function of OSCP is to stabilize the F1FO complex by interacting with $\mathrm{F} 1$, we tried to further probe the association between OSCP loss-of-function and MPTP formation in neurons by disrupting the interaction of OSCP with F1. To this end, we downregulated the $\beta$-subunit, which is the major F1 component that interacts with OSCP in the integral F1FO-ATP synthase $^{30}$ (Supplementary Fig. 5a). Neurons in which the $\beta$-subunit was knockeddown demonstrated significantly suppressed $\mathrm{m} \Delta \Psi$ and reduced ATP generation (Supplementary Fig. $5 b, c)$ in patterns similar to those that we observed in OSCP-deficient neurons (Fig. 2b,c). Importantly, $\beta$-subunit knockdown neurons displayed substantial mPTP activation (Supplementary Fig. 5d). These results indicated that increased mPTP formation is closely associated with F1FO complex disruption, further highlighting a key role of OSCP loss-offunction in triggering mPTP opening. These findings also support previous reports that increased F1 dissociation from the F1FO complex contributes to mPTP formation ${ }^{23,31,32}$.

Mitochondria play a critical role in maintaining synaptic and neuronal function ${ }^{26,33}$. To determine whether loss of OSCP induces synaptic dysfunction, we measured synaptic density by a
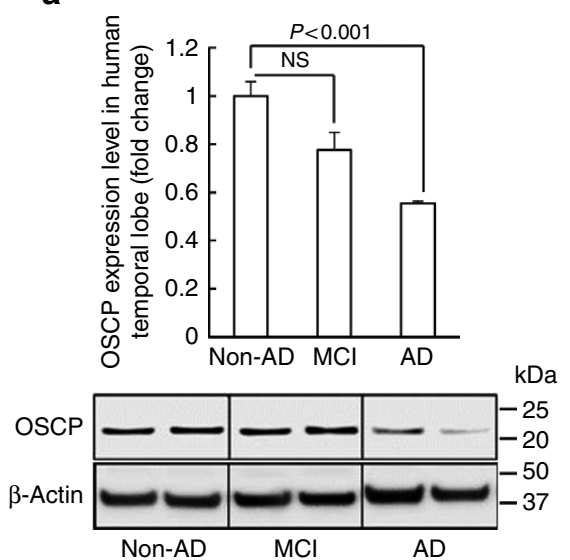

b

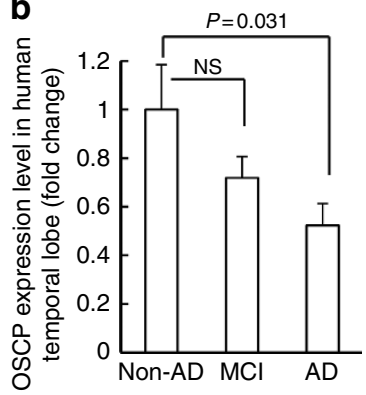

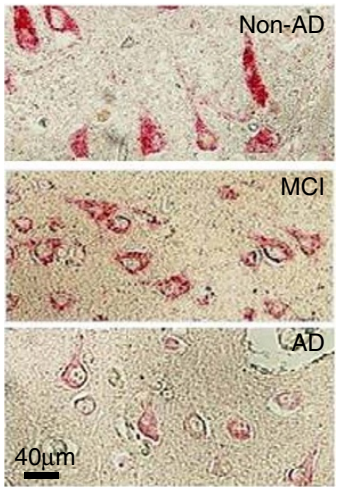

d

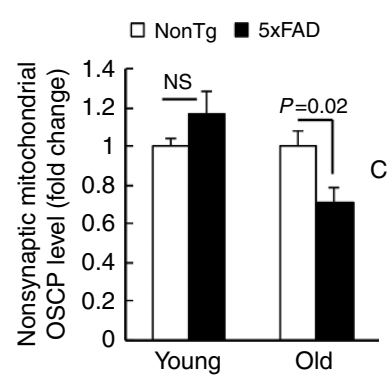

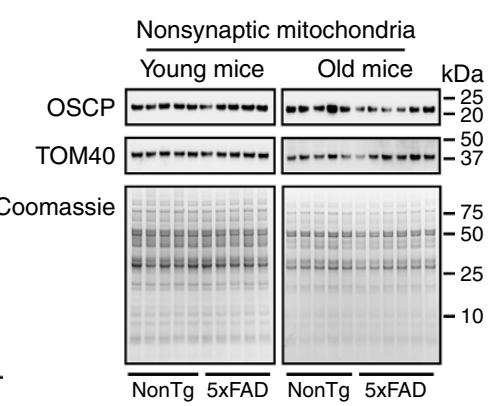

C

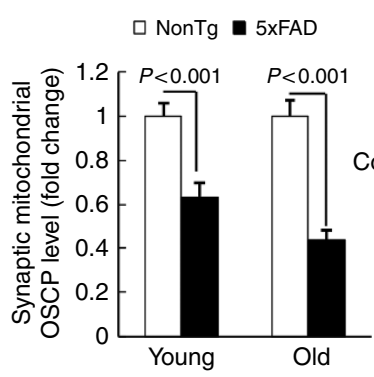

Synaptic mitochondria

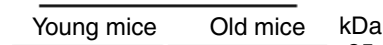

OSCP

TOM40

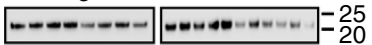
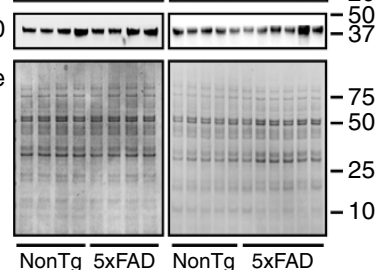
. .

Figure 1 | Loss of OSCP in AD subjects and 5XFAD mouse brain mitochondria. (a) Densitometric quantification of immunoreactive bands of OSCP in protein extracts from the temporal lobes of non- $A D, M C l$ and $A D$ patients. $\beta$-Actin was used to indicate the loading amount of proteins. The lower panel is representative of six non- $A D$, six $\mathrm{MCl}$ and four $A D$ patients. (b) Immunohistochemical staining using anti-OSCP on brain sections from human temporal lobes showed substantial reduction of OSCP in neurons. The right panel shows representative images. Scale bar, $40 \mu \mathrm{m}$. (c,d) Densitometric quantification of OSCP immunoreactive bands shows the age-dependent OSCP reduction in synaptic (c) and nonsynaptic (d) mitochondria from 5xFAD mice. TOM 40 was used to show the enrichment of mitochondrial fractions. Coomassie blue staining was employed to indicate the loading amount of mitochondrial proteins. Five nonTg and five 5xFAD mice at 4 months old and 5 nonTg and 6 5xFA mice at 9 months old were used in the experiments. Error bars represent s.e.m. 
staining for PSD95 and vesicular glutamate transporter 1 (vGlut1). OSCP shRNA-treated neurons showed a significant reduction in synaptic density (Fig. 3a), suggesting a deleterious influence of OSCP loss-associated mitochondrial dysfunction on synaptic function. To directly determine the influence of OSCP loss on synaptic transmission we performed whole-cell voltage-clamp recordings to examine changes in action potentialindependent miniature excitatory postsynaptic currents (mEPSCs). The downregulation of OSCP significantly reduced the average mEPSC amplitude in OSCP knockdown neurons in comparison to their nontarget shRNA-treated controls (Fig. 3b,d,e), without significantly altering mEPSC frequency (Fig. 3b,c). Furthermore, direct stimulation of postsynaptic glutamate receptors by 'puffing' glutamate onto cultured neurons resulted in currents of significantly smaller amplitude in OSCP knockdown neuron (Fig. 3f). Taken together, these data suggest that OSCP loss impairs synaptic function, which parallels the well-documented synaptic dysfunction, and particularly a loss of postsynaptic function, in the pathology of $\mathrm{AD}^{34}$. Energy deprivation, oxidative stress and MPTP formation are hallmarks of mitochondrial defects in AD brains ${ }^{2,5,35,36}$. Synaptic loss and impaired synaptic transmission further characterize AD-related pathological changes ${ }^{34,37-39}$. Therefore, our results suggest that reduced OSCP expression is associated with AD-like mitochondrial dysfunction and the resultant synaptic failure.

Interaction of OSCP with A $\beta$ results in OSCP loss-of-function. $\mathrm{A} \beta$ is a key mediator of $\mathrm{AD}$ and previous studies have shown that $\mathrm{A} \beta$ deposits in AD mitochondria, targeting several mitochondrial proteins ${ }^{2,5,35,40}$. We therefore explored whether OSCP is a binding partner of $\mathrm{A} \beta$ in $\mathrm{AD}$ brains by co-immunoprecipitating temporal lobe protein extracts using anti-OSCP antibody. We detected OSCP/A $\beta$ interaction in AD brains (Fig. 4a), as well as in MCI brains that showed brain $\mathrm{A} \beta$ deposition (Supplementary Fig. 6). In contrast, non-AD brains did not exhibit the OSCP/A $\beta$ complex (Fig. 4a). The OSCP/A $\beta$ complex was not detected in $\mathrm{AD}$ brain tissue when the OSCP antibody was replaced by nonimmune IgG (Fig. 4a), validating the specificity of OSCP/A $\beta$ binding. Similarly, the OSCP/A $\beta$ complex was also found in $5 x F A D$ mice by co-immunoprecipitation (Fig. 4b). Further confocal microscopy studies showed extensive colocalization of OSCP and $A \beta$ in neocortex and hippocampus from $5 x F A D$ mice (Fig. 4c), suggesting an interaction between OSCP and $A \beta$ in vivo. To determine whether OSCP directly interacts with $A \beta$ we next performed an in vitro pull-down assay using glutathione S-transferase (GST)-tagged OSCP as the bait protein. A $\beta 1-42$,
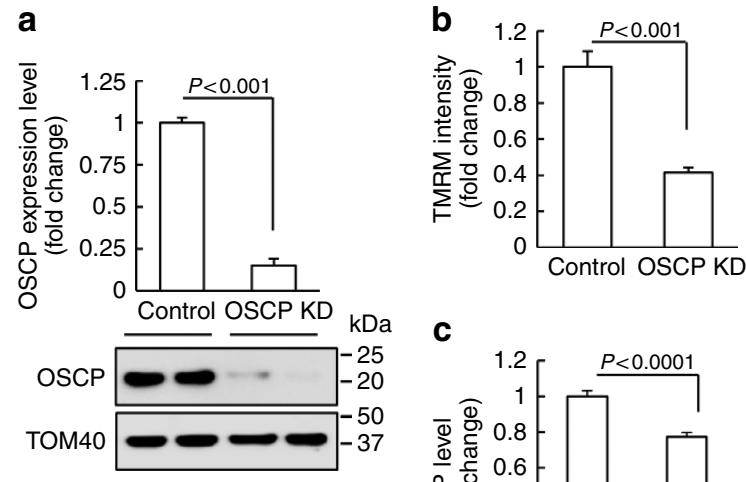

C

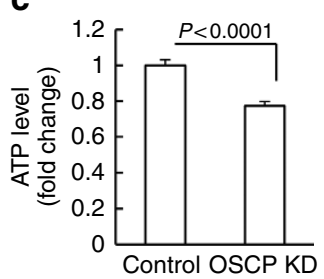

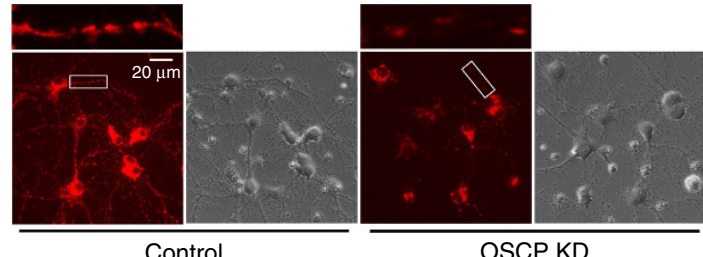

d

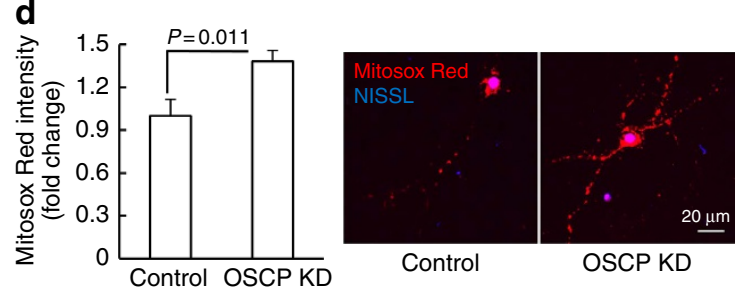

e

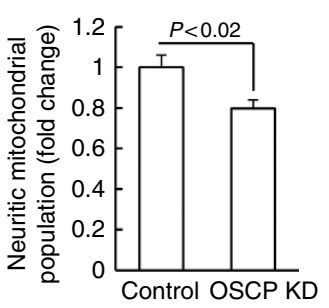

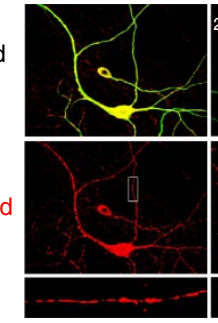

Control

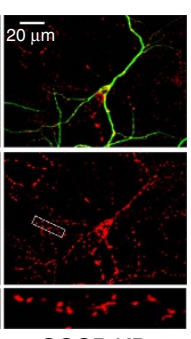

OSCP KD f

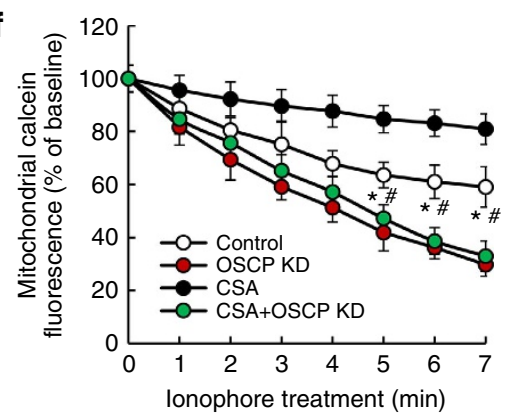

Figure 2 | OSCP deficiency impacts neuronal mitochondrial function. (a) OSCP expression was downregulated in primary cultured mouse neurons by using lentivirus carrying OSCP shRNA (OSCP KD (OSCP knockdown neurons)) and the control neurons were treated by lentivirus carrying nonTarget shRNA. $n=6-9$ samples. The lower panel shows the representative image of immunoreactive bands of OSCP. TOM40 was used as the loading control. (b) OSCP downregulation induced reduction in mitochondrial membrane potential. $n=36-51$ neurons from at least three independent experiments.

The right panel shows representatives of TMRM staining and phase contrast images. Scale bar, $20 \mu \mathrm{m}$. (c) OSCP deficiency also induced decreased neuron ATP production ( $n=13-22$ samples from at least three independent experiments). (d) OSCP deficiency induced increased mitochondrial superoxide levels ( $n=10-15$ neurons from at least three independent experiments). The right panel shows representatives of Mitosox Red staining (red). NISSL (Blue) was used to identify neurons. Scale bar, $20 \mu \mathrm{m}$. (e) Loss of OSCP induced reduction in neuritic mitochondrial population. The right panel shows representative images of MAP2 (green, dendrites) and Mito-Dsred (red, mitochondria) staining. $n=27-43$ neurons from at least three independent experiments. Scale bar, $20 \mu \mathrm{m}$. (f) $\mathrm{mPTP}$ formation demonstrated by the drop in mitochondrial calcein intensity in the exposure of $2 \mu \mathrm{M}$ ionophore. CsA was used at $1 \mu \mathrm{M}$. ${ }^{\star} P<0.01$ versus OSCP KD neurons in the presence or absence of $\mathrm{CsA} .{ }^{\#} P<0.05$ versus control neurons treated by $\mathrm{CsA} . n=5-10$ independent experiments. Error bars represent s.e.m. 
a
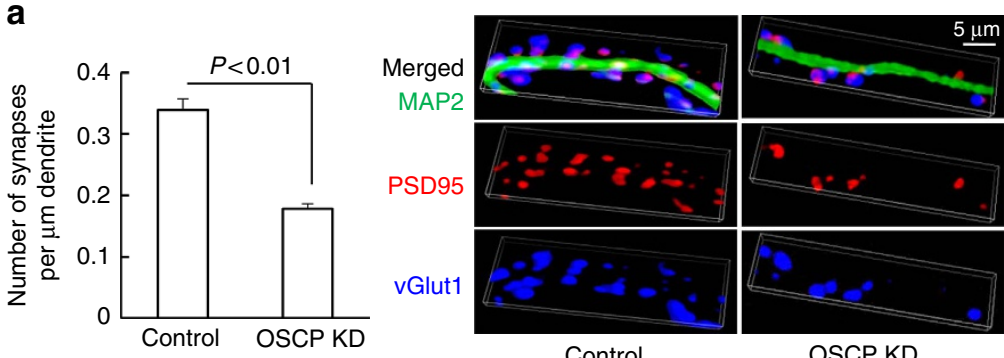

b

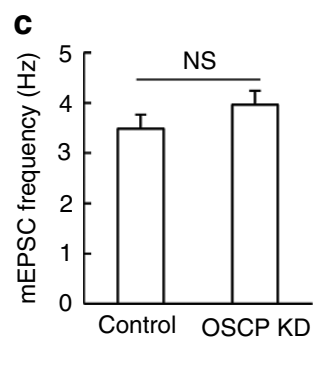

d
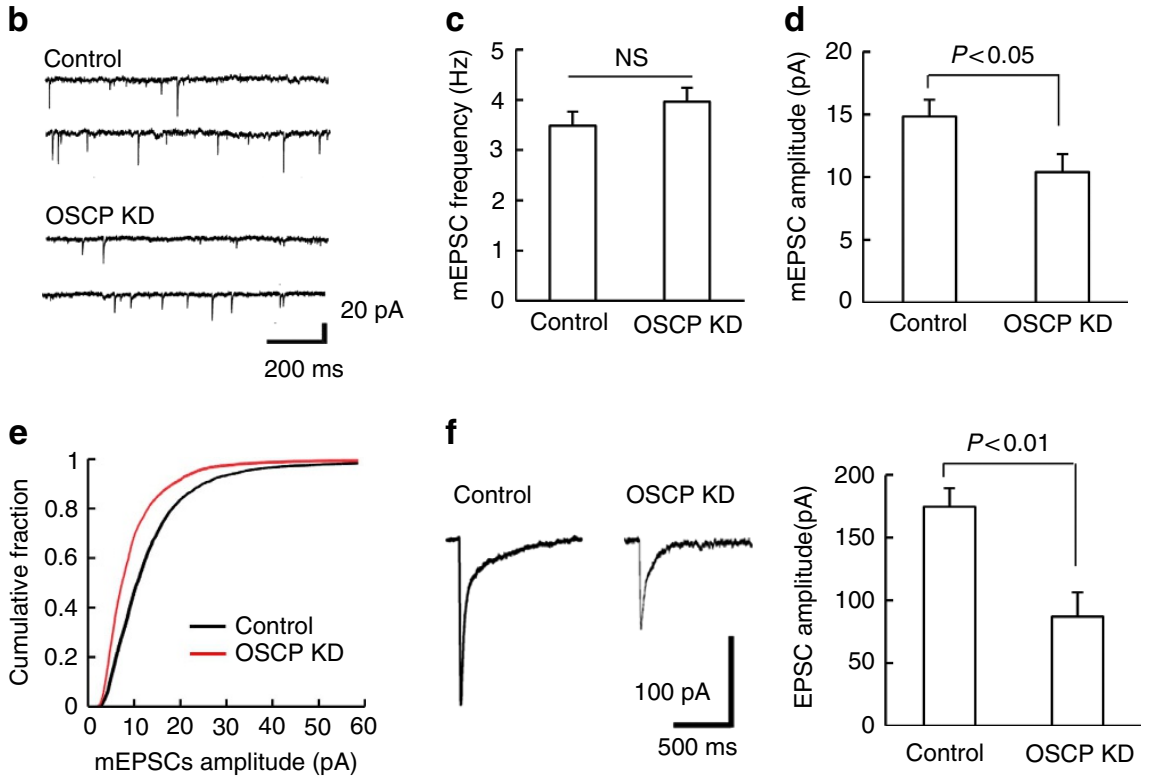

Figure 3 | OSCP deficiency impacts synaptic function. (a) Loss of OSCP induced a decline in synaptic density. The staining of vGlut1 (blue) and PSD95 (red) were used to identify the pre- and post- synaptic components of synapses, respectively. Neuronal dendrites were identified by staining for MAP2 (green). Scale bar, $5 \mu \mathrm{m} . n=27-43$ neurons from at least three independent experiments. (b-e) Loss of OSCP affected mEPSCs. (b) Representative traces of mEPSCs in control (upper panel) and OSCP KD neurons (lower panel). Scale bars represent $200 \mathrm{~ms}$ and 20 pA. Data were collected from 10 control neurons and 7 OSCP knockdown (OSCP KD) neurons from at least three different cultures. (c) Quantitative analysis of mEPSC frequency. (d) Quantitative analysis of mEPSC amplitude. (e) Analysis of the cumulative fraction of mEPSC amplitude distribution. (f) Loss of OSCP reduced the amplitude of EPSCs evoked by puff-application of glutamate. Scale bars represent $500 \mathrm{~ms}$ and $100 \mathrm{pA}$. Data were collected from seven control neurons and eight OSCP KD neurons. The right panel shows quantitative analysis of EPSC amplitude. Error bars represent s.e.m.

but not scrambled A $\beta 1-42$, demonstrated dose-dependent binding with OSCP (Fig. 4d), indicating direct physical interaction of OSCP with $A \beta$.

Physical binding of $A \beta$ could result in functional changes of its target proteins ${ }^{2}$. Therefore, it is important to determine whether the $\mathrm{A} \beta$ interaction influences OSCP function by tethering $\mathrm{F} 1$ via binding with $\alpha$ - and $\beta$-subunits ${ }^{41}$. To address this question, GST-tagged OSCP was pre-incubated with or without A $\beta 1-42$. After washing off unbound $\mathrm{A} \beta 1-42$, we conducted in vitro pull-down assays and found that the binding of OSCP to $\alpha$ - or $\beta$-subunits was significantly reduced by $A \beta 1-42$, suggesting that the interplay between OSCP and A $\beta 1-42$ disrupts the ability of OSCP to bind to the F1 entity (Fig. 4f). To further evaluate the association between $\mathrm{A} \beta$ and impaired OSCP function, we generated deleted forms of OSCP based on the predicted A $\beta$ binding sites on OSCP (Supplementary Fig. $7 \mathrm{a}-\mathrm{c}$ ). We found that the ability of OSCP $\Delta 107-121$ to interact with $\mathrm{A} \beta$ was significantly reduced (Fig. 4e). Furthermore, OSCP $\Delta 107-121$ showed extensive binding with $\alpha$ - or $\beta$-subunits regardless of the presence or absence of $A \beta 1-42$ (Fig. 4f). These results further indicate a direct and specific influence of $\mathrm{A} \beta$ on OSCP, leading to reduced mitochondrial bioenergetics and activated mPTP formation.
To examine the impact of $A \beta$ on OSCP binding to the F1FO-ATP synthase within mitochondrial membranes we exposed brain mitochondria to $A \beta 1-42$. As predicted, co-immunoprecipitation showed OSCP/A $\beta$ complexes in $\mathrm{A} \beta$-exposed mitochondria, indicating that $\mathrm{A} \beta 1-42$ can enter mitochondria and bind to OSCP within the F1FO-ATP synthase (Supplementary Fig. 8a). Next, we examined F1FO-ATP synthase stability by conducting blue-native PAGE (BN-PAGE) ${ }^{42,43}$. Mitochondria were exposed to vehicle-, $\mathrm{A} \beta 1-42-$ or $\mathrm{Ca}^{2+}$. treatment. $\mathrm{Ca}^{2+}$ is thought to disrupt the F1FO complex through $\mathrm{OSCP}^{32}$. A $\beta$ induced a marked increase in $\mathrm{F} 1$ dissociation from the F1FO complex, similar to $\mathrm{Ca}^{2+}$-treatment, while little OSCP was detected in the free F1 (Supplementary Fig. 8b,c). The identification of F1FO dimer, monomer and F1 by $\beta$ subunit blots was further validated by using an antibody against F1 (Supplementary Fig. 8d). Together these data suggest that the $\mathrm{A} \beta$-induced F1FO complex instability is at least partly due to a reduction in OSCP's ability to hold F1 and FO together. As expected, $A \beta 1-42$-treated mitochondria displayed mitochondrial OXPHOS inhibition as evident from the significantly suppressed ATP synthesis (Supplementary Fig. 8e). To dissect the influence of $A \beta$ on mitochondrial OXPHOS enzymes, we measured the enzymatic activities of mitochondrial complexes I through IV and 
a

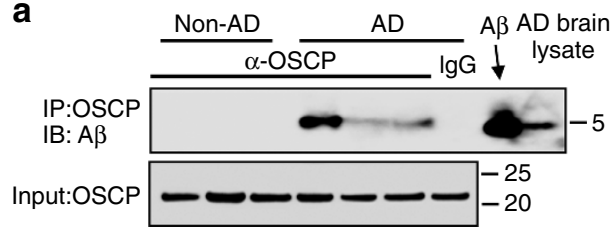

b

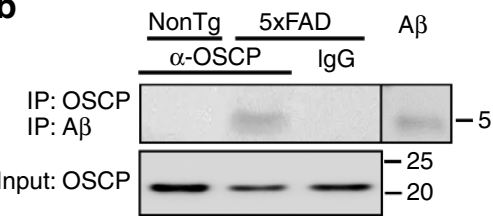

C

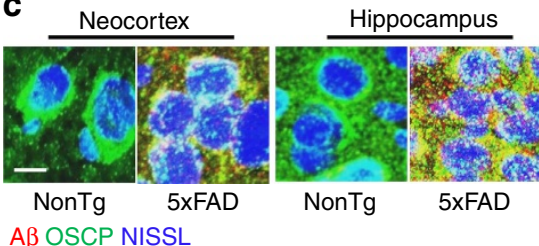

d

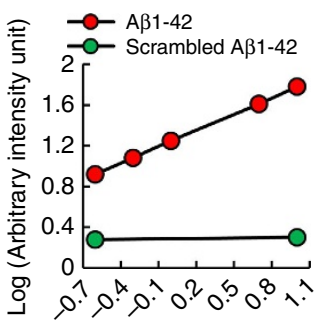

Log $[\mathrm{A} \beta$ concentration $(\mu \mathrm{M})]$

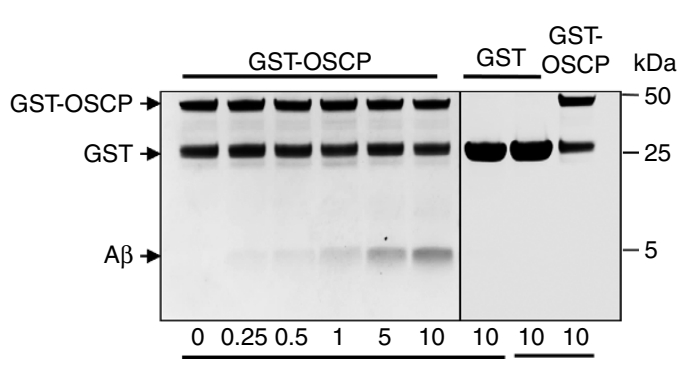

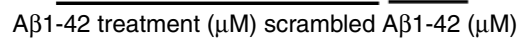

e
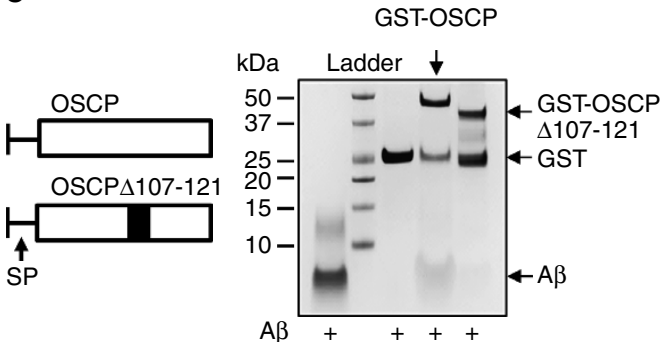

f
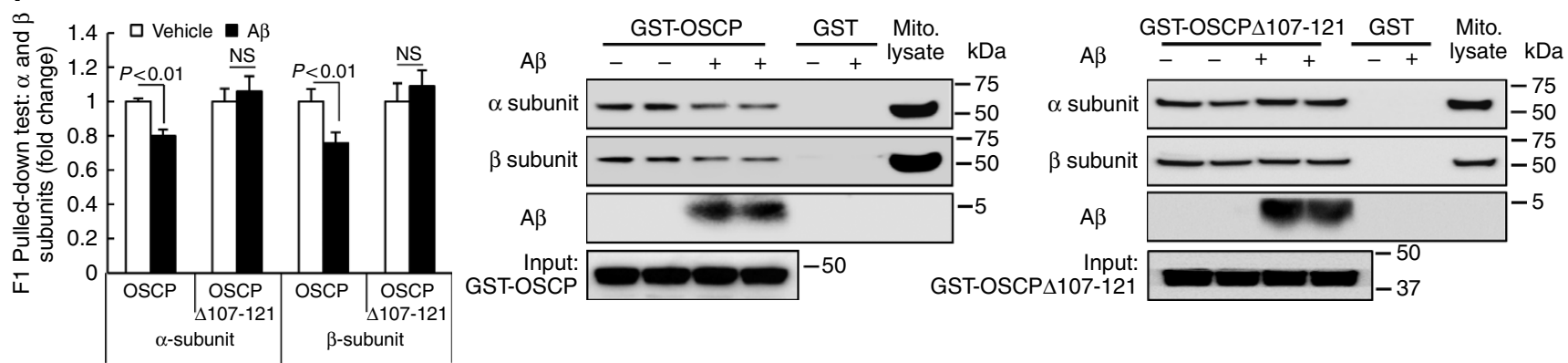

Figure 4 | OSCP/A $\beta$ interactions impact OSCP function in vivo and in vitro. (a) Co-immunoprecipitation of OSCP and $A \beta$ in $A D$ patient temporal lobes. Results shown are representatives from three non-AD and three $A D$ patients. $A \beta 1-42$ peptide and $A D$ brain extracts were used as positive controls for $A \beta$ immunoreactive bands. (b) Co-immunoprecipitation of OSCP and A $\beta$ in 5xFAD mouse neocortex. Results shown are representatives from three mice in each group. $A \beta 1-42$ peptide was used as a positive control for $A \beta$ immunoreactive bands. (c) Colocalization of OSCP (green) and $A \beta$ (red) in neocortex and hippocampus from 5xFAD mice. Neurons were identified by staining for NISSL (blue). Scale bar, $10 \mu \mathrm{m}$. (d) OSCP and A $\beta$ interaction determined by an in vitro pull-down assay. (e) Amino-acid residue numbers are given for mature OSCP protein (with known mitochondrial signalling peptide removed). SP is the mitochondrial signalling peptide. Wild-type OSCP and OSCP $107-121$ were used for pull-down assay. OSCP $\triangle 107-121$ displayed lowered capacity to interact with $A \beta$ compared to wild-type OSCP. (f) In vitro pull-down assay showed that A $\beta 1-42$ suppresses the ability of OSCP, but not OSCP $\triangle 107-121$, to bind $\alpha$ - and $\beta$-subunits as demonstrated by decreased $\alpha$ - and $\beta$-immunoreactive bands. $n=6-10$ samples per group. Error bars represent s.e.m.

of the F1FO-ATP synthase. F1FO-ATP synthase activity was significantly reduced in exogenous $A \beta$-treated mitochondria (Supplementary Fig. 8f); while the activities of mitochondrial complexes I to IV remained unaltered (Supplementary Fig. $8 \mathrm{~g}-\mathrm{j}$ ), suggesting that F1FO-ATP synthase dysfunction confers A $\beta$ toxicity to mitochondrial OXPHOS under the experimental conditions tested.

OSCP aberrations and 5xFAD mouse mitochondrial dysfunction. We hypothesized that if the above effects of OSCP loss and OSCP/A $\beta$ interplay on F1FO-ATP synthase function could be extrapolated to an in vivo setting, $5 x F A D$ mice would display F1FO-ATP synthase dysfunction, particularly in synaptic mitochondria, which demonstrated early and extensive OSCP reduction, as well as $A \beta$ deposition (Supplementary Fig. 9a,b). To address this, we first measured mitochondrial OXPHOS function in $5 \mathrm{xFAD}$ mitochondria. Although nonsynaptic mitochondria from $5 x F A D$ mice also demonstrated an age- and genotypespecific effect (Supplementary Fig. 10a-c), synaptic mitochondria from $5 \mathrm{xFAD}$ mice exhibited an early and marked decrease in the mitochondrial respiratory control ratio, ATP synthesis and F1FO-ATP synthase catalytic activity (Fig. 5a-c). In contrast, mitochondrial complex IV, whose deactivation is thought to be the major OXPHOS defect in $\mathrm{AD}^{8-10}$, exhibited only a relatively mild decrease (Supplementary Fig. 10g,h), again suggesting that F1FO-ATP synthase deregulation contributes to the extensive OXPHOS suppression in 5xFAD mice. Next, we examined F1FO complex proton-flow coupling, which reflects F1FO complex integrity ${ }^{44,45}$. Synaptic mitochondria from $5 x F A D$ mice demonstrated a significant increase in oligomycin-insensitive respiration (that is, uncoupled electron transport, Fig. 5d). This was further supported by a F1FO-ATP synthase coupling assay which showed that $5 x F A D$ synaptic mitochondria had markedly blunted sensitivity to oligomycin-A inhibition (Fig. 5e,f). Such changes were also seen in nonsynaptic mitochondria, but the effect size was considerably smaller (Supplementary Fig. 10d-f). These results imply that F1FO complex destabilization occurs in $5 x F A D$ brain mitochondria. Direct evidence of F1FO complex instability in $5 x F A D$ mitochondria was collected using BN-PAGE. Free F1 was identified by immunoblotting using specific antibodies recognizing the $\beta$ subunit as previously described $^{42}$. We found an age-dependent increase in F1 dissociation (Fig. 5g,h), confirming the destabilization of the F1FO complex in 5xFAD mitochondria. Lastly, because mPTP formation is thought to be a consequence of F1FO complex uncoupling via OSCP deregulation, we measured mPTP 


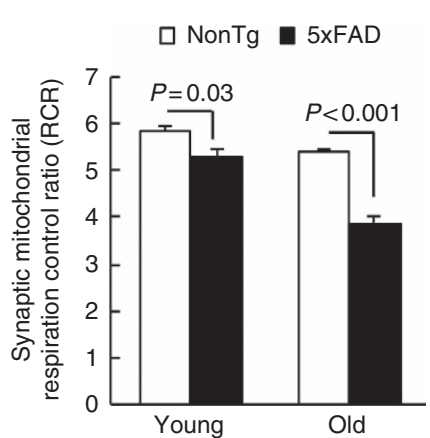

d

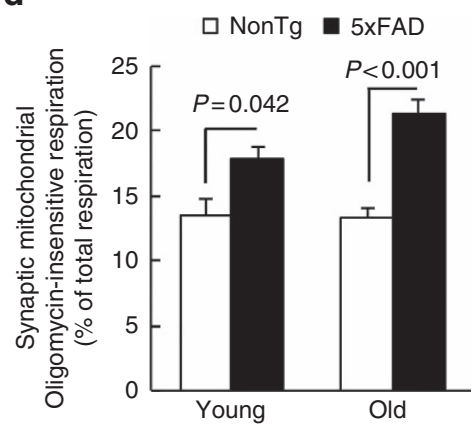

g

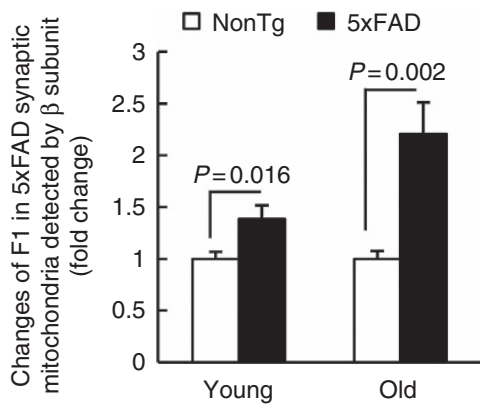

b

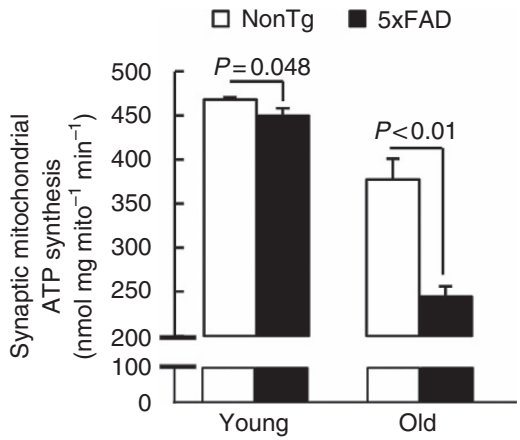

e
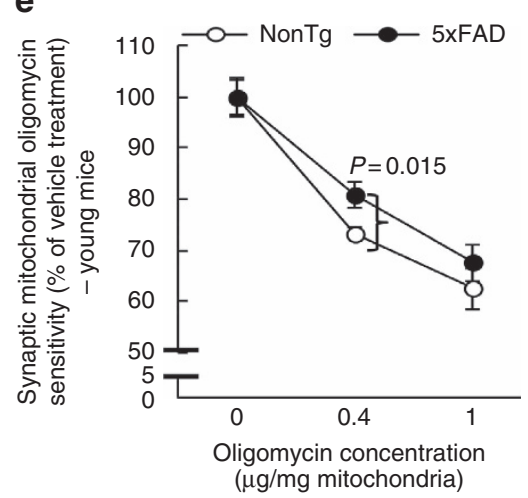

h

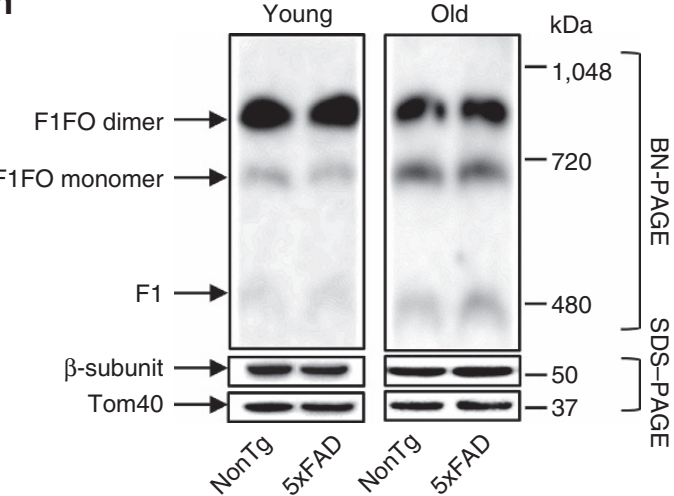

C

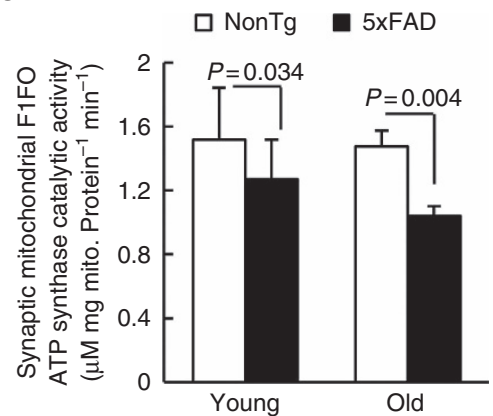

f
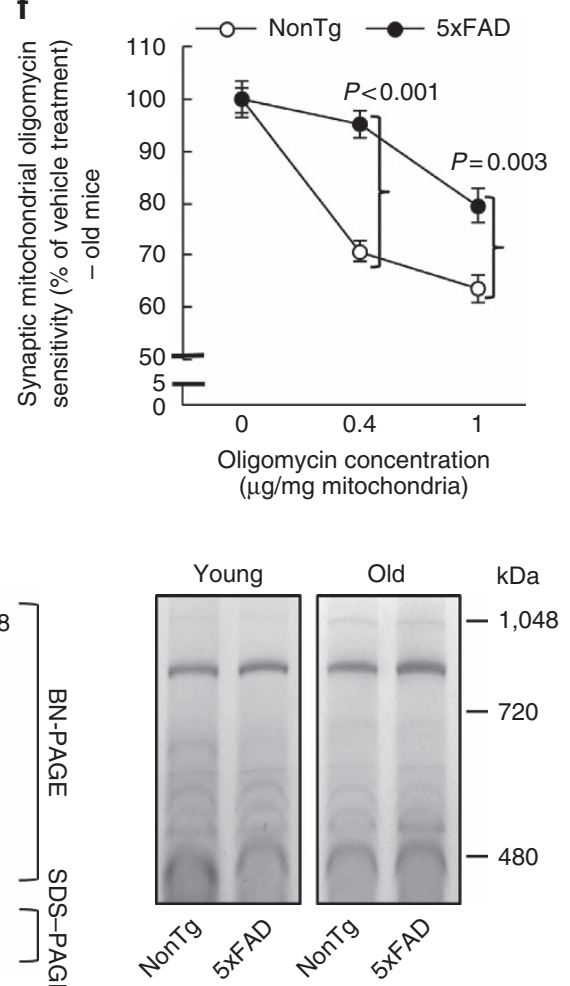

Figure 5 | F1FO-ATP synthase deregulation in 5XFAD mouse synaptic mitochondrial. (a) Synaptic mitochondria from 5xFAD mice demonstrated an age-dependent decrease in their respiratory control ratio (RCR). Six nonTg and 5 xFAD mice at 4 months old and 6 nonTg and 55 xFAD mice at 9 months old were used. (b) Synaptic mitochondria from 5xFAD mice had an age-dependent decrease in ATP synthesis. Six nonTg and $65 \times$ FAD mice at 4 months old and those from six nonTg and seven 5xFAD mice at 9 months old were used in the experiments. (c) Synaptic mitochondria from 5xFAD mice demonstrated an early decrease in the F1FO-ATP synthase catalytic activity at 4 months old which was exacerbated at 9 months old. Five mice of each group at 4 months old and seven nonTg and nine 5xFAD mice at 9 months old were used in the experiment. (d) Age-dependent increase in oligomycin-insensitive respiration of synaptic mitochondria from 5xFAD mice. Six nonTg and five 5xFAD mice at 4 months old, and six nonTg and five 5xFAD mice at 9 months old were used in the experiments. (e,f) Decreased oligomycin sensitivity of synaptic mitochondria from 5xFAD mice at $4(\mathbf{e})$ and 9 months old (f). All data are presented as percentage of the activity of the corresponding vehicle-treated mitochondrial fractions. Six nonTg and five $5 \times F A D$ mice at 4 months old, and seven nonTg and seven 5xFAD mice at 9 months old were used in the experiments. (g,h) Increased F1 dissociation in synaptic mitochondria from 5xFAD mice. (g) The analysis of free F1. (h) The left panel is the representative of images collected from seven nonTg and six $5 \times$ FAD mice at 4 months old, and six nonTg and six 5xFAD mice at 9 months old. F1 was determined by probing with anti- $\beta$ subunit antibody and the molecular weight of the bands. The same amount of samples was used for SDS-PAGE and Tom 40 and $\beta$ subunit were detected to show the loading amount of mitochondrial proteins. The right panel is the coomassie blue staining before immunoblotting to indicate the loading amount of samples. Error bars represent s.e.m.

formation susceptibility and found that $5 x F A D$ synaptic mitochondria exhibited a significantly increased response to $\mathrm{Ca}^{2+}$-induced mitochondrial swelling (Supplementary Fig. 10i), which is in agreement with our previous findings ${ }^{33}$. Therefore, these data show a strong correlation between OSCP deregulation and mitochondrial dysfunction in AD-relevant pathophysiological settings.
Protection of OSCP restoration on A $\beta$-exposed mouse neurons. To further address the role of OSCP deregulation for the induction of mitochondrial dysfunction in an $A \beta$-rich environment we examined whether the $A \beta$-mediated mitochondrial dysfunction can be attenuated by OSCP restoration. Therefore, we overexpressed OSCP in mouse neurons, aiming to restore OSCP levels and to dilute the OSCP/A $\beta$ interaction which would 
be expected to protect OSCP function against $\mathrm{A} \beta$ toxicity. The control and OSCP overexpressing (OSCP OE) neurons were exposed to a treatment with oligomeric $A \beta 1-42$. By immunoblotting and immunostaining (Fig. 6a,b), we found that A $\beta$-mediated OSCP reduction in control neurons was significantly ameliorated by OSCP upregulation. Further mitochondrial functional assays showed that OSCP overexpression substantially attenuated the decreased $\mathrm{m} \Delta \Psi$, lowered ATP production and decreased neuritic mitochondrial population as well as sensitized mPTP formation in A $\beta$-treated neurons (Fig. 6c-f). Notably, OSCP overexpression by itself did not significantly alter the expression level of CypD (Supplementary Fig. 11). To investigate the specific protection of OSCP restoration against $A \beta$ toxicity on neuronal mitochondria we overexpressed the F1FO-ATP synthase $\beta$-subunit, which forms the catalytic core of the F1FO-ATP synthase. We then treated the control and $\beta$-subunit-overexpressing ( $\beta$-subunit $\mathrm{OE}$ ) neurons with $A \beta$. A $\beta$ treatment did not induce detectable changes in the expression levels of $\beta$-subunit in either control or $\beta$-subunit OE neurons when compared with their vehicle-treated counterparts
(Supplementary Fig. 12a), which supports our findings of the unaltered $\beta$-subunit levels in $\mathrm{AD}$ subjects (Supplementary Fig. 1a,b) as well as in 5xFAD mice (Supplementary Fig. 3a,c). However, further experiments showed that the overexpression of $\beta$-subunit did not demonstrate significant protection against $A \beta$ induced $\mathrm{m} \Delta \Psi$ collapse, ATP reduction or decrease in neuritic mitochondrial population, as well as mPTP activation (Supplementary Fig. 12b-e), further supporting a specific protective effect of OSCP restoration in AD-related conditions.

We used exogenous $A \beta$ treatment to mimic the high level of $\mathrm{A} \beta$ over-production in $\mathrm{AD}$ subjects; however, it is not clear whether OSCP restoration has similar protective effects in neurons that generate endogenous $A \beta$. To address this question we used primary neuron cultures from $5 x F A D$ mice. In comparison to nonTg neurons, cultured $5 \times \mathrm{xFAD}$ neurons demonstrated a significant loss of OSCP expression, which was prevented by OSCP overexpression (Supplementary Fig. 13a). Importantly, OSCP overexpression in $5 x F A D$ neurons exhibited protective effects on $\mathrm{m} \Delta \Psi$, ATP production and neuritic mitochondrial population, as well as $\mathrm{mPTP}$ formation a

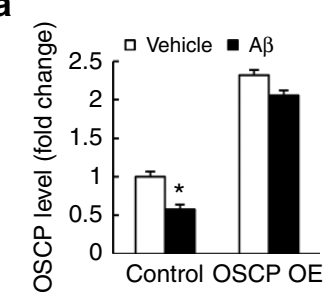

b

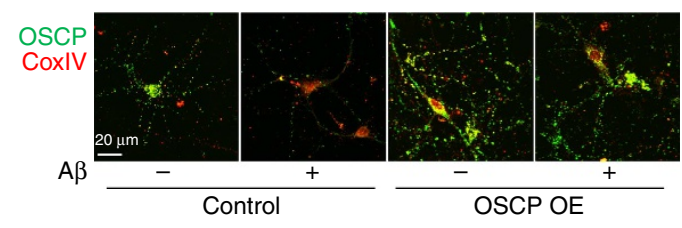

e

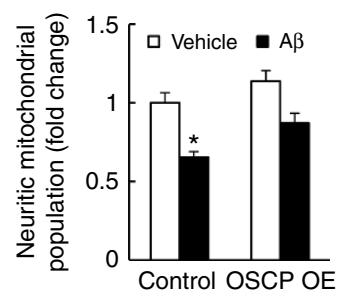

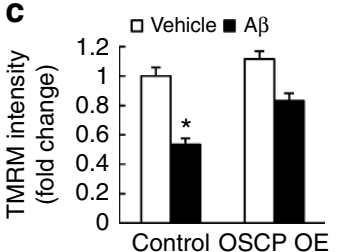

d

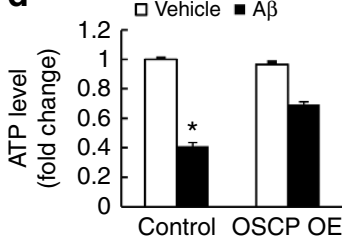

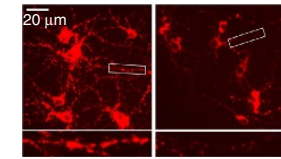
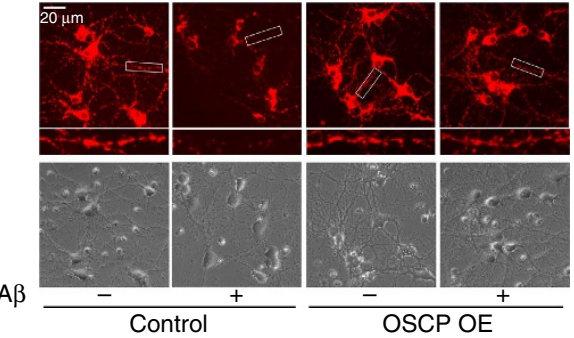

f
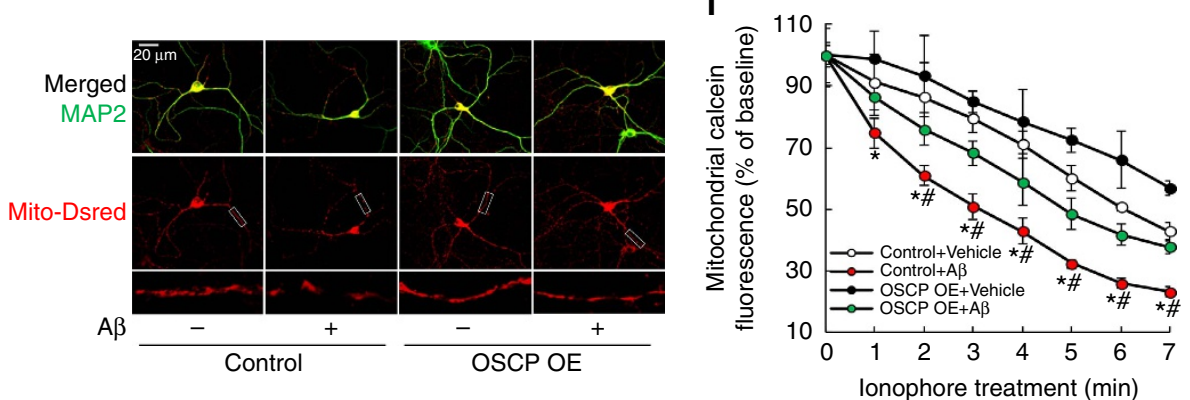

Figure 6 | OSCP overexpression ameliorates A $\boldsymbol{\beta}$-induced mitochondrial dysfunction in mouse neurons. Mouse cortical neurons were exposed to $500 \mathrm{nM}$ oligomeric $\mathrm{A} \beta 1-42$ for $24 \mathrm{~h}$. (a) Attenuated oligomeric A $\beta 1$-42-induced OSCP reduction by OSCP overexpression. Western blot images show OSCP expression level in mouse primary neurons which is representative of seven samples in each group. (b) Shows immunofluorescent staining of OSCP (green) in primary cultured neurons. COXIV (red) was used to determine the localization of OSCP in mitochondria. ${ }^{\star} P<0.01$ versus other groups. Scale bar, $20 \mu \mathrm{m}$. (c) OSCP overexpression protected mitochondrial membrane potential against oligomeric A $\beta 1-42$ toxicity. The upper panel of representative

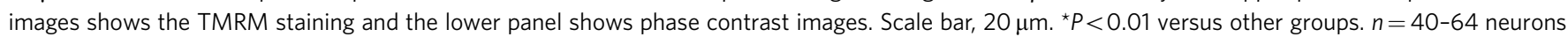
from at least three independent experiments. (d) OSCP overexpression protected neuron ATP reduction against oligomeric $A \beta 1-42$ toxicity. ${ }^{\star} P<0.01$ versus other groups. $n=12$ samples of each group from at least three independent experiments. (e) Attenuated $A \beta$-induced reduction in neuritic mitochondrial population by OSCP overexpression. ${ }^{\star} P<0.01$ versus other groups. $n=22-27$ neurons from at least three independent experiments. The upper panel of representative images shows the merged staining of MAP2 (green, dendrite) and Mito-Dsred (red, mitochondria). Scale bar, $20 \mu$ m. The middle panel shows Mito-Dsred staining and the lowest panel shows enlarged images from the indicated areas. (f) Ameliorated A $\beta$-sensitized mPTP formation by OSCP overexpression. lonophore $(2 \mu \mathrm{M})$ was used to trigger mPTP formation. ${ }^{\star} P<0.05$ versus vehicle-treated control and OSCP OE neurons. ${ }^{\#} P<0.05$ versus $A \beta$-treated OSCP OE neurons. $n=4-6$ independent experiment. Control refers to neurons infected with control lentivirus carrying backbone vector. OSCP OE refers to neurons infected with lentivirus carrying OSCP cDNA. Error bars represent s.e.m. 
(Supplementary Fig. 13b-e) against endogenous $\mathrm{A} \beta$ toxicity in similar patterns as we observed in neurons treated with exogenous $A \beta$, confirming the protective effects of OSCP restoration against $A \beta$ toxicity.

Given the crucial role of mitochondria in sustaining synaptic transmission and plasticity ${ }^{46}$, and the attenuation of mitochondrial defects by OSCP overexpression, we next examined whether OSCP restoration also protects synaptic function against $A \beta$ toxicity. Control and OSCP OE neurons were exposed to vehicle- or oligomeric $A \beta 1-42$-treatment before the synaptic density was analysed using immunofluorescent staining of PSD95 and vGlut1. Oligomeric A $\beta 1-42$-treated control neurons displayed a significant reduction in synaptic density in comparison to control neurons receiving vehicle treatment (Fig. 7a). In sharp contrast, the $A \beta$-induced synaptic loss was significantly ameliorated by OSCP overexpression (Fig. 7a). OSCP overexpression by itself did not affect baseline levels of synaptic density (Fig. 7a). In addition, OSCP overexpression protected against the reduction in mEPSC amplitude induced by $A \beta$ toxicity (Fig. $7 \mathrm{~b}, \mathrm{~d}, \mathrm{e}$ ). The relatively preserved mEPSC frequency of $A \beta$-treated neurons (Fig. $7 \mathrm{~b}, \mathrm{c}$ ) may reflect distinct time-dependent changes in pre- and post-synaptic function during acute $\mathrm{A} \beta$ treatment ${ }^{47}$, and/or may result from presynaptic calcium accumulation due to $\mathrm{MPTP}$ activation $^{48}$, which could affect asynchronous neurotransmitter release and thus mEPSC frequency ${ }^{49}$. Moreover, in OSCP overexpressing neurons the postsynaptic response to glutamate stimulation was indistinguishable from that in control neurons even in the presence of exogenous $A \beta$ (Fig. 7f). Taken together, our results suggest that the restoration of OSCP protects neuronal mitochondrial and synaptic function from $\mathrm{A} \beta$ toxicity.

Protection of OSCP restoration on A $\beta$-exposed human neurons. Because $\mathrm{AD}$ is a human disease, it is of considerable interest to know whether OSCP restoration confers similar protection to mitochondria in $A \beta$-exposed human neurons. Therefore we derived human neurons from human neural stem cells (Supplementary Fig. 14) and treated them with oligomeric A $\beta 1-42$. Immunobloting for OSCP levels and co-immunoprecipitation of OSCP and $A \beta$ revealed that the OSCP loss and the formation of the OSCP/A $\beta$ complex (Fig. 8a,b) observed in AD individuals were mirrored in $A \beta$-treated human neurons. Next, OSCP was overexpressed in human neurons (Fig. 8c). Control and OSCP OE human neurons were then exposed to oligomeric $\mathrm{A} \beta 1-42$ at $500 \mathrm{nM}$ and then processed for our assays of mitochondrial
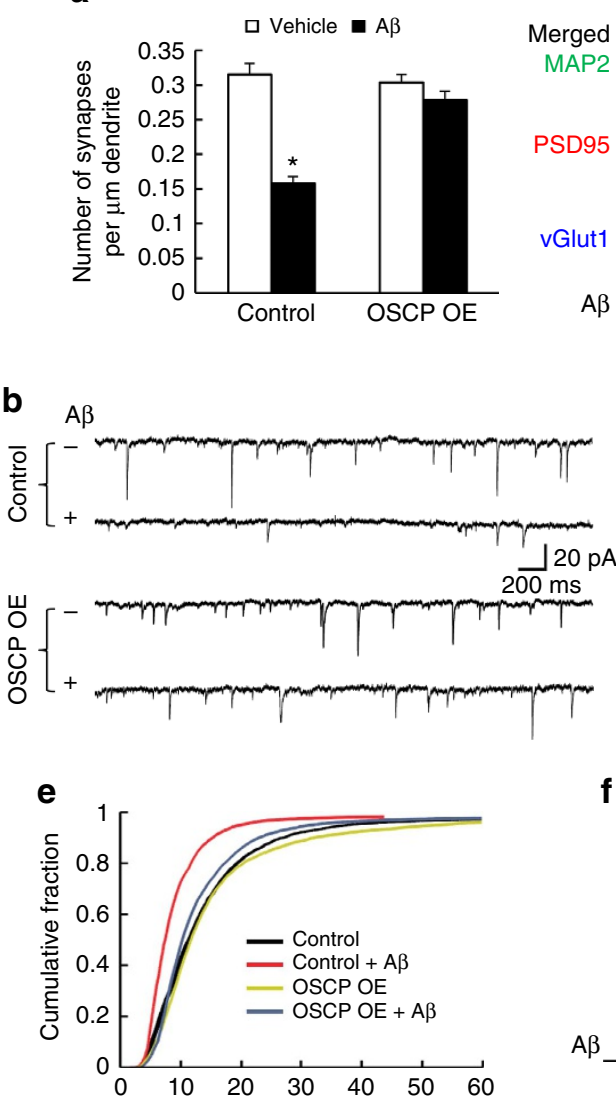

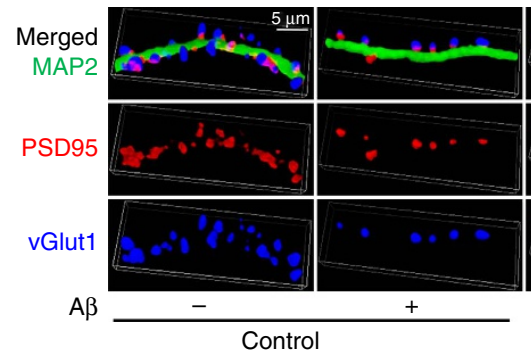

C

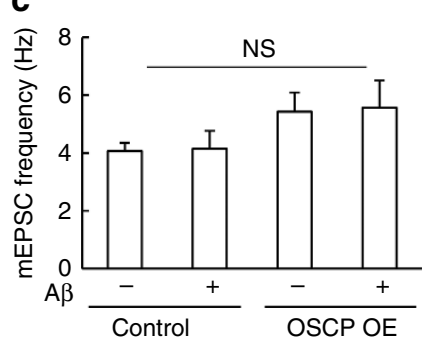

f

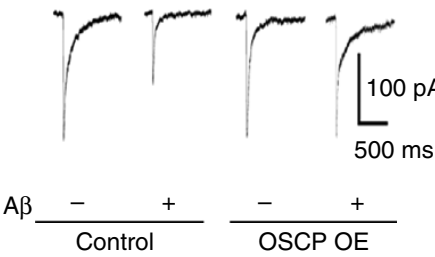

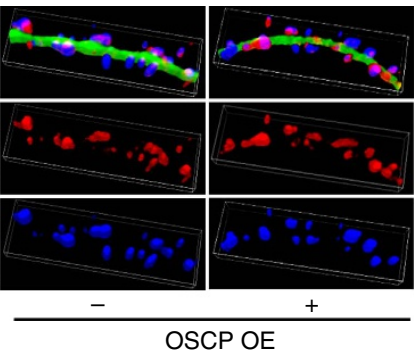

d
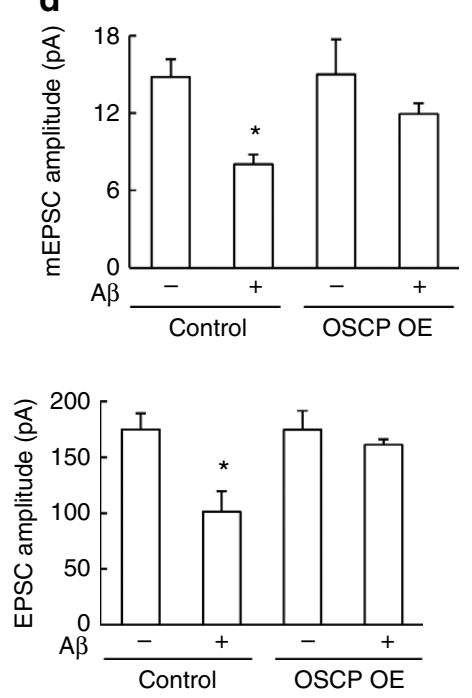

Figure 7 | OSCP overexpression protects mouse neurons against A $\boldsymbol{\beta}$ induced synaptic dysfunction. (a) Attenuated A $\beta$-induced synaptic density reduction in OSCP OE neurons. ${ }^{\star} P<0.01$ versus other groups. $n=26-43$ neurons collected from at least three independent experiments. Synapses were visualized by the staining for vGlut1 (Blue) and PSD95 (red) to identify the pre- and postsynaptic components of synapses, respectively. Neuronal dendrites were determined by MAP2 (green). Scale bar, $5 \mu \mathrm{m}$. (b-e) OSCP overexpression protected mEPSCs from A $\beta$ toxicity. (b) Representative traces of mEPSC recordings from control and OSCP OE neurons in the presence or absence of A 3 . Scale bars represent $200 \mathrm{~ms}$ and $20 \mathrm{pA}$. The data were collected from 10 vehicle-treated control neurons, 8 A $\beta$-treated control neurons, 7 vehicle-treated OSCP OE neurons and 8 A $\beta$-treated OSCP OE neurons. (c) Quantitative analysis of mEPSC frequency. (d) Quantitative analysis of mEPSC amplitude. ${ }^{\star} P<0.05$ versus other groups. (e) The cumulative fraction of mEPSC amplitude distribution. (f) Overexpression of OSCP prevented the reduction in amplitude of glutamate-evoked EPSCs that results from A $\beta$ toxicity in control neurons. Scale bars represent $500 \mathrm{~ms}$ and $100 \mathrm{pA}$. Data were collected from seven vehicle-treated control neurons, nine $A \beta$-treated control neurons, five vehicle-treated OSCP OE neurons and seven A $\beta$-treated OSCP OE neurons. Error bars represent s.e.m. 

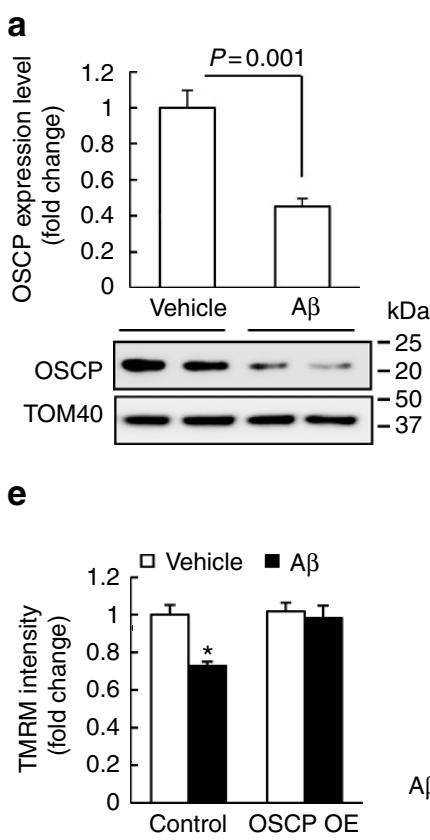

$\mathbf{f}$

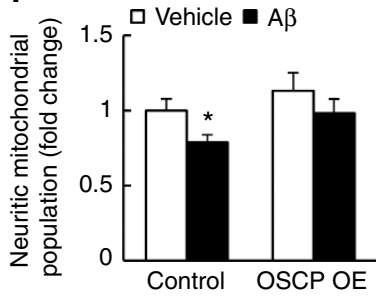

b

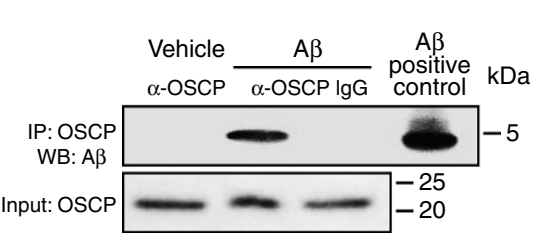

C

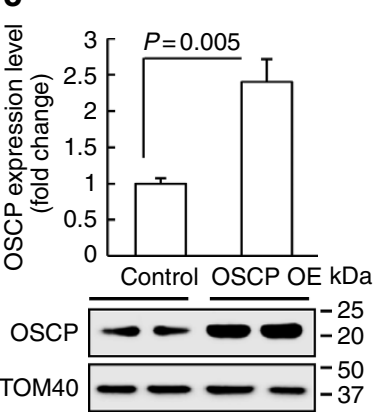

d

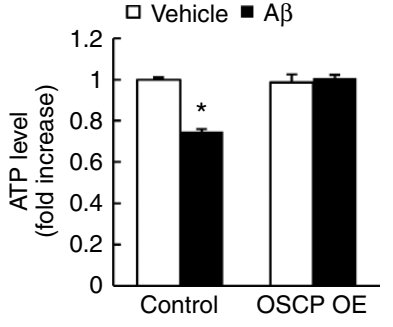

$A \beta$
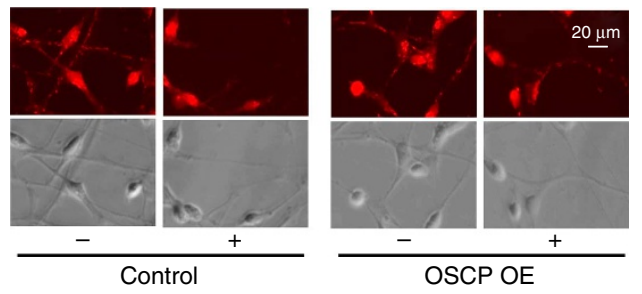

MAP2

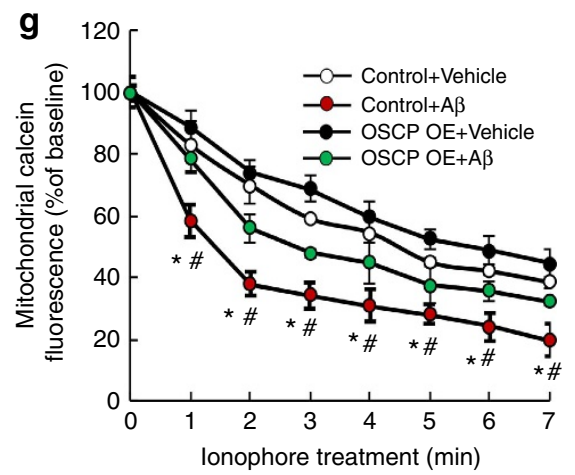

lonophore treatment $(\mathrm{min})$

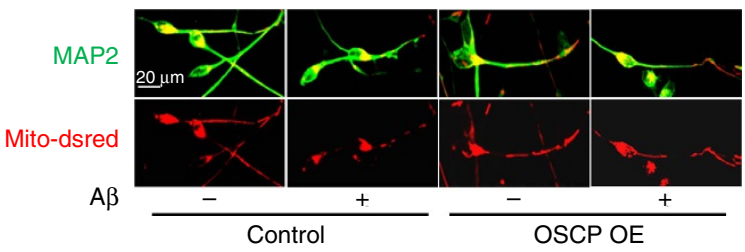

Figure 8 | OSCP overexpression ameliorates $\mathbf{A} \boldsymbol{\beta}$-induced mitochondrial dysfunction in human neurons. (a) Human neurons were exposed to $500 \mathrm{nM}$ oligomeric A $\beta 1-42$ for 4 days, then subjected to immunoblotting detection of OSCP levels. A $\beta$ induced a significant reduction in OSCP expression level. The lower panel shows representative immunoblotting images. Tom40 was used as the loading control. $n=4-5$ samples of each group. (b) Co-immunoprecipitation of OSCP and A $\beta$ in A $\beta$-treated human neurons. (c) Significantly increased OSCP expression in the OSCP overexpressing neurons. The lower panel shows representative immunoblotting images. Tom 40 was used as the loading control. $n=4$ samples of each group.

(d) Preserved ATP production by OSCP overexpression. ${ }^{\star} P<0.01$ versus other groups. $n=6-10$ samples of each group. (e) Preserved mitochondrial membrane potential by OSCP overexpression. ${ }^{\star} P<0.01$ versus other groups. $n=17-23$ neurons from at least 3 time independent experiments. Right panels are representative images of TMRM staining (upper row) and phase contrast (lower row). Scale bar, $20 \mu \mathrm{m}$. (f) Preserved neuritic mitochondrial population by OSCP OE. ${ }^{\star} P<0.05$ versus other groups. Right panels are representative images. Dendrites were determined by the staining of MAP2 (green); and mitochondria were identified by mito-Dsred (red). Scale bar, $20 \mu \mathrm{m}$. (g) Attenuated A $\beta$-sensitized mPTP formation by OSCP overexpression. Ionophore $(2 \mu \mathrm{M})$ was used to trigger mPTP formation. ${ }^{\star} P<0.05$ versus vehicle-treated control and OSCP OE neurons. ${ }^{\#} P<0.05$ versus A $\beta$-treated OSCP OE neurons. $n=4-7$ independent experiments. Error bars represent s.e.m.

function. Our results showed that $\mathrm{A} \beta$-induced mitochondrial dysfunctions in ATP production, $\mathrm{m} \Delta \Psi$ and neuritic mitochondrial population, as well as mPTP formation (Fig. 8d-g) were substantially reduced by OSCP overexpression in human neurons. Moreover, the overexpression of OSCP in human neurons did not have a detectable influence on baseline levels of the assayed parameters in comparison to control neurons (Fig. 8d-g), which further supports our observations in OSCP OE mouse neurons.

\section{Discussion}

In this study, we find that OSCP loss and OSCP/A $\beta$ interaction constitute the major OSCP alterations in the brains from $\mathrm{AD}$ patients and 5xFAD mice. Among the major subunits of F1FOATP synthase, OSCP is selectively decreased in the brains of AD subjects and $5 \mathrm{xFAD}$ mice. Moreover, the early decrease of OSCP expression in synaptic mitochondria from $5 x F A D$ mice indicates that synaptic mitochondria are more vulnerable to $\mathrm{A} \beta$-induced mitochondrial alterations. This reveals a novel form of synaptic mitochondrial stress in $\mathrm{AD}$ and supports a causative role of synaptic mitochondrial dysfunction in the development of early synaptic dysfunction in the disease ${ }^{33}$. Another critical finding of this study is the interaction of OSCP and $A \beta$. In recent years, the accumulation of $A \beta$ in mitochondria, and particularly in synaptic mitochondria, has received considerable attention ${ }^{33}$. $A \beta$ is probably transported via a translocase in the outer mitochondrial membrane $e^{50}$ and impacts mitochondrial function via multiple pathways, including the interaction of $A \beta$ with several mitochondrial proteins such as $\mathrm{CypD}^{5}$, Amyloid betabinding alcohol dehydrogenase $e^{35}$ and dynamin-like protein 1 (ref. 40). Our finding of significant interplay between OSCP and $A \beta$ furthers our understanding of the intracellular influence of $A \beta$ toxicity in $\mathrm{AD}$. Indeed, given the extensive $\mathrm{A} \beta$ accumulation in mitochondria, we cannot exclude the possibility that mitochondrial $A \beta$ may bind to other proteins embedded in the inner mitochondrial membrane or to other subunits of mitochondrial F1FO-ATP synthase, which could also be involved in compromised F1FO-ATP synthase function in AD. In view of 


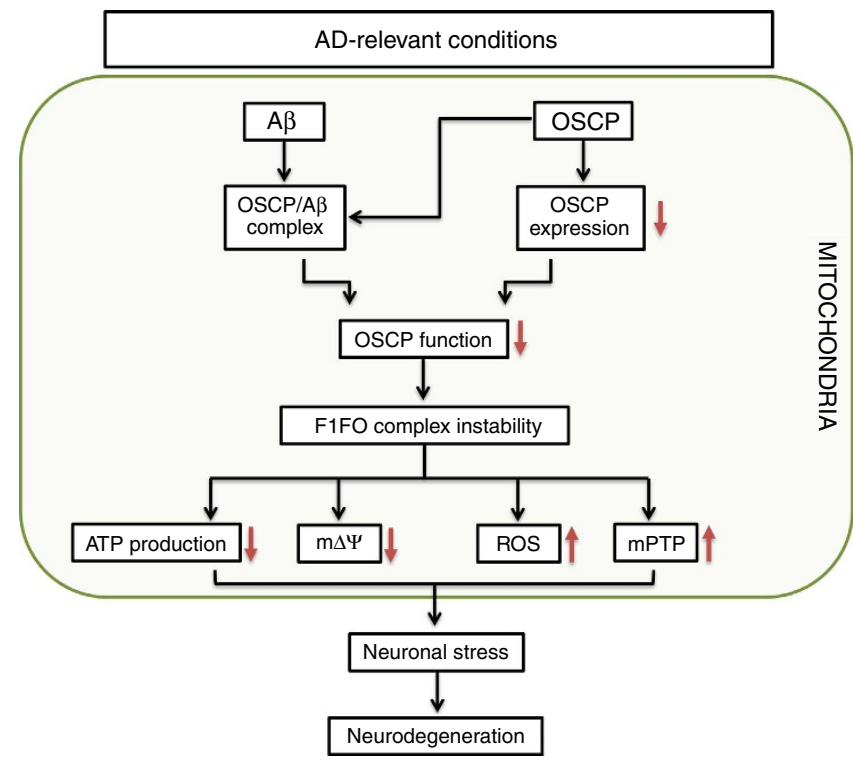

Figure 9 | Schematic summary. With the progress of $A D$, brain mitochondria gradually undergo OSCP loss and $A \beta$ accumulation in $A D$-relevant conditions. OSCP loss and OSCP/A $\beta$ interaction impair OSCP function to keep F1FO complex integrity. This leads to severe mitochondrial dysfunction, including decreased ATP production, collapsed mitochondrial membrane potential, and increased ROS production and release, as well as the activation of mitochondrial permeability transition pore formation. Such mitochondrial deregulation results in dampened neuronal function and eventually neuronal death.

the critical role that the $\beta$-subunit plays for the catalytic functions of F1FO-ATP synthase, we examined whether the $\beta$-subunit is a binding partner of $A \beta$. Our data (not shown) suggest that the $\beta$-subunit is not a likely binding partner of $A \beta$. Future investigation will explore whether $A \beta$ may affect other mitochondrial proteins that contribute to mitochondrial F1FOATP synthase dysfunction, as well as MPTP activation in AD.

Our findings of reduced OSCP levels and the interaction of $A \beta$ with OSCP, which impair mitochondrial function in AD-related pathological settings, implicate that $A \beta$ toxicity induces OSCP aberrations, leading to mitochondrial F1FO-ATP synthase dysfunction in AD-sensitive brain areas. However, increasing evidence suggests that $\mathrm{AD}$ is a systemic disease. In addition to $A D$-affected brain areas, $A \beta$ is detected in circulating blood ${ }^{51}$ and platelets ${ }^{52,53}$, as well as in other tissues such as intestine and kidney ${ }^{54}$. The systemic distribution of $A \beta$ raises the possibility of $\mathrm{A} \beta$ interaction with OSCP in cells outside the nervous system, which may potentially cause mitochondrial dysfunction. Moreover, in view of the complexity of mitochondrial abnormalities in $\mathrm{AD}$ our findings of OSCP aberrations may only provide one mechanism for systemic mitochondrial dysfunction. Therefore, it will be important for future studies to evaluate the impact of OSCP alterations in $\mathrm{AD}$ on a systems level.

Suppressed mitochondrial OXPHOS is a hallmark of mitochondrial defects in $\mathrm{AD}^{2,3}$. However, the detailed mechanisms of this mitochondrial deficit in $\mathrm{AD}$ are not fully understood. It is well accepted that $\mathrm{AD}$ is accompanied by pronounced changes in the function of mitochondrial complex IV (refs 8-10). Indeed, we have found a significant decrease of complex IV activity in $5 \mathrm{xFAD}$ neuronal mitochondria when comparing it to the corresponding complex IV activity in nonTg mice. However, the contribution of complex IV dysfunction to ATP deficiency in $\mathrm{AD}$ is still controversial ${ }^{11-13}$. Given the redundant capacity of complex IV in mitochondria, it has been argued that the slight decrease in complex IV activity does not explain severe ATP deprivation in $A D^{11}$, suggesting that other mitochondrial OXPHOS enzymes also become dysfunctional. F1FO-ATP synthase is a critical OXPHOS enzyme synthesizing ATP ${ }^{18,19}$. To date, our knowledge on the functional state of mitochondrial F1FO-ATP synthase in AD is extremely limited. Earlier studies suggested that F1FO-ATP synthase is not involved in AD because the F1FO-ATP synthase enzyme activity in the brains or platelets of $\mathrm{AD}$ patients ${ }^{55}$ appeared to be unchanged, and increased enzymatic activity was observed in platelets from probable $\mathrm{AD}$ subjects $^{56}$. However, this concept has been challenged in recent years by evidence that showed alterations of this enzyme in AD-sensitive brain regions, and particularly in neurons ${ }^{14-17}$. Indeed, as we show in this study, synaptic mitochondria are more vulnerable to $A \beta$-induced F1FO-ATP synthase dysfunction in $5 x F A D$ mice, whereas nonsynaptic mitochondria are relatively preserved. Further, we show that F1FO complex uncoupling is a prominent defect in $5 \mathrm{xFAD}$ mouse brain mitochondria. The coupling state of this enzyme in $\mathrm{AD}$, however, was largely overlooked in previous studies, which failed to fully evaluate F1FO-ATP synthase deregulation in AD. Therefore, our results showing that F1FO-ATP synthase deregulation is associated with suppressed neuronal mitochondrial OXPHOS efficacy provide a novel mitochondrial mechanism of neuronal stress in $\mathrm{AD}$ and impact our current understanding of mitochondrial OXPHOS deficits in this disease.

The molecular identity of mPTP has long been a critical scientific issue ${ }^{23}$. Recently, uncoupled mitochondrial F1FO-ATP synthase was identified as the molecular basis of $\mathrm{mPTP}^{23}$. Specifically, the prevalent model proposes that OSCP is the binding target of CypD, which is a non-pore forming regulator of $\mathrm{mPTP}$ opening. The interaction of CypD with OSCP triggers the instability of the F1FO complex, thus resulting in the dissociation of F1 and eventually the formation of a nonselective leak channel within $c$ rings ${ }^{22,24}$. These findings have suggested a key role of OSCP deregulation in the induction of MPTP. However, whether this model applies to neuronal mitochondria has not been comprehensively investigated. By downregulating OSCP in neuronal mitochondria, we found substantially sensitized MPTP formation. Importantly, this OSCP loss-associated mPTP activation is indispensable of the function of CypD, thus serving as strong evidence of the role of OSCP deregulation for inducing $\mathrm{MPTP}$ formation in neurons. Our in vivo results further link mPTP over-activation to OSCP aberrations in AD-relevant pathological settings. Sensitized MPTP formation is well documented as a key mitochondrial dysfunction in AD. Our previous studies have shown that mPTP blockade by CypD depletion protects mitochondrial and neuronal stress in a mouse model of AD, suggesting that $\mathrm{mPTP}$ regulation could be a therapeutic strategy for $\mathrm{AD}$ treatment ${ }^{5,26}$. The physiological function of mPTP has been noticed in recent years ${ }^{57-59}$. Molkentin and colleagues ${ }^{57}$ found increased susceptibility of heart failure in CypD-deficient mice outlining the risks associated with inhibiting the physiological functions of MPTP and CypD. Therefore, it would be preferable to develop a strategy that can reduce $\mathrm{mPTP}$ over-activation under pathological states like AD while preserving $\mathrm{mPTP}$ function under normal physiological conditions. In this study, we found that OSCP restoration reduces $\mathrm{A} \beta$-induced $\mathrm{mPTP}$ over-activation without having a detectable influence on baseline mPTP formation. Therefore, protecting OSCP seems to be a promising avenue for the regulation of $\mathrm{MPTP}$ for AD treatment.

Lastly, mitochondrial dysfunction has been identified as a causative factor of synaptic failure in $\mathrm{AD}$. Therefore, supporting mitochondrial function seems to be a viable strategy to protect synaptic strength and plasticity to delay the cognitive decline in 
$\mathrm{AD}$ patients ${ }^{1,2}$. We found that loss of OSCP induces synaptic dysfunction, which was paralleled by reduced mitochondrial function. Notably, the restoration of OSCP mitigated A $\beta$-mediated mitochondrial dysfunction and further preserved synaptic function as evidenced by our measures of synaptic density and synaptic transmission. Therefore, our results confirm the deleterious impact of mitochondrial injury on synaptic function and further highlight the therapeutic role of OSCP restoration for the protection of synaptic strength to ameliorate cognitive impairments in $\mathrm{AD}$.

In summary, we have uncovered a novel mechanism of mitochondrial dysfunction mediated via OSCP disruption in an $\mathrm{AD}$-relevant pathological setting. Moreover, our results indicate a role of mitochondrial F1FO-ATP synthase deregulation in the development of $\mathrm{AD}$ mitochondrial defects that has long been overlooked. However, other factors may also contribute to mitochondrial F1FO-ATP synthase dysfunction in AD. For example, a previous study has shown that the $\alpha$-subunit interacts with extracellular domain of amyloid beta precursor protein (APP) and $A \beta$ on neuronal surface $A T P a s e^{60}$. In addition, oxidative stress disrupts ATP synthase activity ${ }^{19}$, and ATP synthase $\alpha$-subunit O-GlcNAcylation is decreased in AD-related conditions $^{61}$. These changes may also potentially affect mitochondrial F1FO-ATP synthase. Thus future studies need to fully explore mitochondrial F1FO-ATP synthase dysfunction in AD. Nevertheless, the most parsimonious interpretation of our findings is that F1FO-ATP synthase deregulation via OSCP links mitochondrial defects to $\mathrm{AD}$ (Fig. 9) and constitutes a novel target for $\mathrm{AD}$ therapy.

\begin{abstract}
Methods
Mice. Animal studies were approved by the University of Texas at Dallas Institutional Animal Care and Use Committee (IACUC) and were performed in accordance with the National Institutes of Health guidelines for animal care. $5 \mathrm{xFAD}$ mice overexpress a human form of mAPP-bearing mutations (SwFlLon) and PSEN1 mutations (M146L and L286V) linked to familial AD.

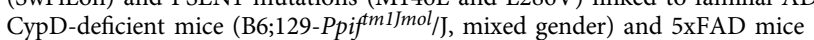
(B6SJL-Tg(APPSwFlLon, PSEN1 ${ }^{\star}$ M146L ${ }^{*}$ L286V) 6799Vas/Mmjax, mixed gender) were obtained from Jackson Laboratory. Mice were allocated randomly to experimental groups for the various experimental measurements based on genotyping. Four and nine months old nonTg and $5 \times \mathrm{xFAD}$ mice of mixed genders were used in the experiments. Day 0-1 nonTg, 5xFAD and CypD-deficient pups were used for primary neuron culture. The investigators performing the experiments did not select the mice allocation. The genotype of all the transgenic animals were double checked by performing PCR and/or dot blots before the experiments. Mice with wrong genotyping were excluded. The number of mice was determined by our previous data and power calculation to ensure the minimal numbers of mice as needed were used in the experiments.
\end{abstract}

Human samples. Frozen brain samples and paraffin-embedded brain slices were requested from UT Southwestern Medical Center ADC Neuropathology Core, supported by ADC grant (AG12300) under a protocol approved by The UT Southwestern Medical Center with informed consent from all subjects and the study adhered to the Declaration of Helsinki principles.

Mitochondria preparation. Synaptic and nonsynaptic mitochondria were isolated from tissue as previously described, brain tissues were homogenized in ice cold isolation buffer ( $225 \mathrm{mM}$ mannitol, $75 \mathrm{mM}$ sucrose, $2 \mathrm{mM} \mathrm{K2PO4,} \mathrm{0.1 \%} \mathrm{BSA,}$ $5 \mathrm{mM}$ Hepes, $1 \mathrm{mM}$ EGTA (pH 7.2)) with a Dounce homogenizer (Wheaton). The resultant homogenate was centrifuged at $1,300 \mathrm{~g}$ for $3 \mathrm{~min}$, and the supernatant was layered on a $3 \times 2-\mathrm{ml}$ discontinuous gradient of 15,23 and $40 \%$ ( $\mathrm{vol} / \mathrm{vol}$ ) Percoll and centrifuged at 34,000 g for 8 min (flying time) on Beckman Coulter ultracentrifuge (Optima XPN-90 Ultracentrifuge). After centrifugation, the interface between 15 and $23 \%$ (Band containing synaptosomes) was collected. Additionally, the interface between 23 and 40\% (containing nonsynaptic mitochondria) was removed and collected. The fractions were then resuspended in isolation buffer containing $0.02 \%$ digitonin and incubated on ice for $5 \mathrm{~min}$. The suspensions were then centrifuged at $16,500 \mathrm{~g}$ for $15 \mathrm{~min}$. The resulting loose pellets were washed for a second time by a centrifugation at $8,000 \mathrm{~g}$ for $10 \mathrm{~min}$. Pellets were collected and resuspended in isolation buffer. Percoll density gradient centrifugation was performed as described above for a second time. The interface between 23 and $40 \%$ (mitochondria released from synaptosomes) was collected and resuspended in isolation buffer to centrifuge at $16,500 \mathrm{~g}$ for $15 \mathrm{~min}$. The resultant pellet was resuspended in isolation buffer followed by a centrifugation at $8,000 \mathrm{~g}$ for $10 \mathrm{~min}$. The final synaptic mitochondrial pellet was resuspended in isolation buffer and stored on ice during experiments. Protein concentration was determined using the Bio-Rad DC protein assay (Bio-Rad Laboratories).

ATP synthase catalytic activity assay. ATP synthase activity was measured spectrophotometrically using NADH-linked, ATP-regenerating system ${ }^{62}$. Briefly, mitochondria of appropriate amount were placed in ATP synthase Assay buffer (100 mM Tris-HCl (pH 7.4), $2 \mathrm{mM} \mathrm{MgCl} 2,50 \mathrm{mM} \mathrm{KCl}, 0.2 \mathrm{mM}$ EDTA, $0.23 \mathrm{mM}$ $\mathrm{NADH}$ and $1 \mathrm{mM}$ phosphoenolpyruvate). The reaction was triggered by the addition of $0.4 \mathrm{M}$ ATP-Mg and recorded on a spectrophotometer (Ultrospect 2100 , Amersham Biosciences) at OD340 nm for a total of $600 \mathrm{~s}$ at 10 -s intervals.

ATP synthase coupling assay. Mitochondrial fractions $(15 \mu \mathrm{g})$ were placed in ATP synthase assay buffer ( $100 \mathrm{mM}$ Tris- $\mathrm{HCl}(\mathrm{pH} 7.4), 2 \mathrm{mM} \mathrm{MgCl}_{2}, 50 \mathrm{mM} \mathrm{KCl}$, $0.2 \mathrm{mM}$ EDTA, $0.23 \mathrm{mM} \mathrm{NADH}$ and $1 \mathrm{mM}$ phosphoenolpyruvate) in the presence of oligomycin-A at $0,0.4$ and $1 \mu$ g oligomycin-A per mg mitochondrial protein for $15 \mathrm{~min}$ at room temperature. After the incubation, ATP synthase activity was measured spectrophotometrically using NADH-linked, ATP-regenerating system.

ATP synthesis assay. Aliquots of mitochondria were analysed by using the ATP Luminescent assay kit (Abcam). Briefly, mitochondria were placed in isolation buffer ( $225 \mathrm{mM}$ mannitol, $75 \mathrm{mM}$ sucrose, $2 \mathrm{mM} \mathrm{K}_{2} \mathrm{PO}_{4}, 0.1 \%$ BSA, $5 \mathrm{mM}$ HEPES, $1 \mathrm{mM}$ EGTA (pH 7.2)). Mitochondria were energized with $5 \mathrm{mM}$ glutamate/malate and ATP synthesis was induced with the injection of $500 \mu \mathrm{M}$ ADP. ATP determination was accomplished following the manufacturer's instructions.

Mitochondrial respiration assays. Purified mitochondria were energized by glutamate $(5 \mathrm{mM})$ and malate $(5 \mathrm{mM})$ and subjected to respiration assays on a Clark electrode. Oxygen consumption was triggered by the addition of ADP. The mitochondrial respiratory control ratio was defined as the ratio of State III respiration/State IV respiration. To measure oligomycin-insensitive respiration, mitochondria were energized by glutamate $(5 \mathrm{mM})$ and malate $(5 \mathrm{mM})$ and oxygen consumption was triggered by the addition of ADP as described above. ADP-triggered respiration was collected as total respiration. Oligomycin $(5 \mu \mathrm{M})$ was then added and the after-oligomycin respiration was collected. The oligomycin-insensitive respiration was calculated as the percentage of after-oligomycin respiration in total respiration.

Mitochondrial swelling assay. Mitochondria were suspended in $0.5 \mathrm{ml}$ of swelling assay buffer $(50 \mu \mathrm{g}$ of mitochondrial protein, $150 \mathrm{mM} \mathrm{KCl}, 5 \mathrm{mM}$ HEPES, $2 \mathrm{mM} \mathrm{K}_{2} \mathrm{HPO}_{4}, 5 \mathrm{mM}$ glutamate ( $\left.\mathrm{pH} 7.3\right)$ ). Mitochondrial swelling was triggered by the addition of calcium ( $500 \mathrm{nmol} \mathrm{mg}^{-1}$ of protein). Swelling was observed by immediately and continuously recording changes at OD $540 \mathrm{~nm}$ by using a spectrophotometer (Ultrospect 2100, Amersham Biosciences) for a total of $600 \mathrm{~s}$ at $10-\mathrm{s}$ intervals.

Immunoblotting analysis. Samples were prepared in sample loading buffer (50 mM Tris-HCl pH 6.8, 2\% SDS, $10 \%$ glycerol, $1 \% \beta$-mercaptoethanol, $12.5 \mathrm{mM}$ EDTA and $0.02 \%$ bromophenol blue) and proteins were separated by SDS-PAGE (10 or $12 \%$ Bis-Tris gel; Life Technologies), and then transferred to a PVDF membrane for blotting (Bio-Rad Laboratories). After blocking in TBS buffer (20 mM Tris- $\mathrm{HCl}, 150 \mathrm{mM}$ sodium chloride) containing $5 \%$ (wt/vol) nonfat dry milk for $1 \mathrm{~h}$ at room temperature, the membrane was then incubated and gently shaken overnight at $4{ }^{\circ} \mathrm{C}$ with primary antibodies. This was followed by incubation with the corresponding secondary antibody for $1 \mathrm{~h}$ at room temperature. The following antibodies were used: mouse monoclonal anti ATP5A (Life Technologies, \#459240, 1:5,000), rabbit polyclonal anti ATP5B (Santa Cruz, \#sc-33618,1:2,000) and mouse monoclonal anti-OSCP (Santa Cruz, \#sc-365162, 1:5,000), goat polyclonal anti subunit c (Santa Cruz, \#sc-132636, 1:1,000), goat polyclonal anti- subunit b (Santa Cruz, \#sc-162552, 1:500), goat polyclonal anti subunit a (Proteintech, \#55313-1-AP, 1:5,000), rabbit polyclonal anti-TOM40 (Santa Cruz, \#sc-11414, 1:1,000), rabbit polyclonal anti-A $\beta$ (CST, \#8243, 1:5,000), mouse anti- $\beta$ actin (Sigma-Aldrich, \#5441, 1:10,000), mouse anti-Cyclophilin D (Millipore, \#AP1035, 1:3,000), rabbit anti-VDAC (CST, \#4661, 1:5,000), Goat anti-mouse IgG HRP conjugated and goat anti-rabbit IgG HRP conjugated (Life Technologies, \#626520 and 656120, 1:2,000-8,000). Images were collected on Bio-Rad Chemidoc Imaging System. Image J software (National Institutes of Health) was used to analyse the scanned blots and to quantify protein signal intensity.

Co-immunoprecipitation. Mitochondria from cerebral cortices of transgenic mice or human subjects were suspended in buffer $\left(500 \mu \mathrm{g} \mathrm{ml}^{-1}, 50 \mathrm{mM}\right.$ Tris, $150 \mathrm{mM} \mathrm{NaCl}, 1 \mathrm{mM}$ EDTA, protease inhibitors (Calbiochem, set V, EDTA free), $0.1 \% \mathrm{NP}-40, \mathrm{pH} 7.5$ ) and then subjected to five freeze-thaw cycles, followed by centrifugation at $12,000 \mathrm{~g}$ for $10 \mathrm{~min}$ at $4{ }^{\circ} \mathrm{C}$. We immunoprecipitated the resulting supernatant with mouse antibody to OSCP (Santa Cruz, \#sc-365162, $0.5 \mu \mathrm{g}$ IgG per 
$100 \mu \mathrm{g}$ protein) at $4{ }^{\circ} \mathrm{C}$ overnight, followed by an incubation with protein $\mathrm{A} / \mathrm{G}$ agarose (Pierce) for $2 \mathrm{~h}$ at room temperature. Nonimmune IgG $(0.5 \mu \mathrm{g} \operatorname{IgG}$ per $100 \mu \mathrm{g}$ protein) was used as negative control. We subjected the resultant immunoprecipitant to immunoblotting with antibody to A $\beta$ (CST, \#8243, 1:4,000).

Immunostaining analysis. For brain slices, slices were deparaffinized and rehydrated before they were subjected to antigen retrieval by boiling in citric acid buffer for $15 \mathrm{~min}$. For cultured neurons, neurons were fixed in $4 \%$ paraformaldehyde for $30 \mathrm{~min}$ at $37^{\circ} \mathrm{C}$. After blocking (5\% Goat serum, $0.3 \%$ Triton X-100, PBS), the slices were incubated with antibodies against A $\beta$ (CST, \#8243,1:1,000), COXIV (CST, \#4844, 1:300), MAP2 (Sigma-Aldrich, \#M4403, 1:300), OSCP (Santa Cruz, \#sc-365162, 1:200) and with Nissl staining (Life Technologies, \#N21479, 1:200) in combination or separately. After washing in PBS, the slices were probed with appropriate secondary antibodies including Alexa Fluor@ 488 and Alexa Fluor@ 594 (Life Technologies, 1:500). Images were collected on a Nikon confocal microscope.

GST-OSCP pulldowns. GST pulldown was performed according to manufacturer's protocol (Pierce). Briefly, the human OSCP cDNA (Gene Name: ATP5O. NCBI Gene ID: 539) or deleted form of OSCP cDNA was transformed into BL21 (DE3) pLysS Escherichia coli (Promega) using the pGEX-4t-1 plasmid (GE Healthcare). After transformation and selection a single colony was chosen for PCR to verify positive transformation. After overnight growth and induction by IPTG (Sigma-Aldrich), bacteria were pelleted and then were lysed by sonication in $1 \times$ PBS containing $0.2 \mathrm{mM}$ PMSF and $100 \mu \mathrm{g} \mathrm{ml}^{-1}$ lysozyme. After sonication bacterial debris was removed by centrifugation at $12,000 \mathrm{~g}$ for $15 \mathrm{~min}$ at $4^{\circ} \mathrm{C}$. Supernatant was collected and incubated with glutathione agarose high-capacity, high-performance resin (Pierce) for $2 \mathrm{~h}$. Glutathione beads were then washed and incubated overnight at $4{ }^{\circ} \mathrm{C}$ with mitochondria lysates or $\mathrm{A} \beta$ peptide. After washing, protein was eluted from the beads and separated by SDS-PAGE. Immunoblotting or coomassie staining was performed to visualize results.

Protein-protein predictions. HADDOCK (high ambiguity-driven proteinprotein docking ${ }^{63}$ was used to predict protein-protein interaction between ATP5O (PDB\# 2WSS; s chain) and amyloid beta (PDB\# 1Z0Q). The modelling was performed in ambiguous interaction restraints with CPORT'S active and passive constraints. Models with $z$-scores below -1.4 were selected and amino acids on OSCP within $4 \AA$ of amyloid beta were determined using $\mathrm{PyMOL}^{64}$.

Deletion constructs. Regions of interest were determined by both HADDOCK modelling and based on literature analysis ${ }^{65,66}$. Four OSCP deletions constructs were made; OSCP $\Delta 1-18$, OSCP $\Delta 168-190$, OSCP $\Delta 1-18 ; 168-190$ and OSCP $\Delta 107-121$. Theses constructs were generated via PCR and inserted into a GST-bearing vector (pGEX-4t-1). The vector was subsequently transformed into BL21(DE3) pLysS E. coli (Promega). After transformation and selection of a single colony was chosen for PCR to verify positive transformation. Protein products were obtained via IPTG induction and purification with glutathione agarose high-capacity, high-performance resin (Pierce).

Oligomeric A $\beta$ preparation. $A \beta 1-42$ peptide (GenicBio) was diluted in 1,1,1,3,3,3,-hexafluoro-2-propanol to $1 \mathrm{mM}$ using a glass gas-tight Hamilton syrings with a Teflon plunger. The clear solution was then aliquoted in microcentrifuge tubes, and it was dried by vaporation in the fume hood. Peptide film was diluted in DMSO to $5 \mathrm{mM}$ and sonicated for $10 \mathrm{~min}$ in bath sonicator. The peptide solution was resuspended in cold HAM'S F-12 to $100 \mu \mathrm{M}$ and immediately vortexed for $30 \mathrm{~s}$. The solution was then incubated at $4{ }^{\circ} \mathrm{C}$ for $24 \mathrm{~h}$.

ELISA assay for mitochondrial $\mathbf{A} \beta$ measurement. $A \beta$ level in mitochondrial fractions was measured by using human $A \beta 1-40$ and $A \beta 1-42$ ELISA kits (Life Technologies) following the manufacturer's instructions.

Mouse neuron culture. Cortices or hippocampi were dissected from day 0-1 pups in cold Hank's buffer (without $\mathrm{Ca} 2+$ and $\mathrm{Mg} 2+$ ), dissociated with $0.05 \%$ trysin at $37^{\circ} \mathrm{C}$ for $15 \mathrm{~min}$ followed by 10 times trituration in ice cold neurobasal A medium. Cells were then passed through $40 \mu \mathrm{m}$ mesh cell strainer (Corning) and centrifuged for $2 \mathrm{~min}$ at $200 \mathrm{~g}$. The pellet was gently resuspended in neuron culture medium (neurobasal A with $2 \% \mathrm{~B} 27$ supplement, $0.5 \mathrm{mM} \mathrm{L}$-glutamine, $50 \mathrm{U} \mathrm{ml}^{-1}$ penicillin, and $50 \mu \mathrm{g} \mathrm{ml}^{-1}$ streptomycin) and plated on poly-D-lysine- (Sigma-Aldrich) coated culture plates (Corning) or Lab-Tek chamber slides (Nunc, 177445) with an appropriate density.

Human neural stem cell culture and neural cell differentiation. Human neural stem cells (StemPro Neural Stem Cells, \#A10509-01, Life Technologies) were cultured and differentiated into neurons as manufacturer's instruction. Differentiated neurons were determined by the morphology as well as the staining of neuronal-specific marker $\beta I I I$ tubulin using an antibody against $\beta I I I$ tubulin
(CST, \#D71G9, 1:300) followed by a secondary antibody of Alexa Fluor@ 594 (Life Technology, \#A-11037, 1:500).

OSCP knockdown in mouse primary neurons. Lentivirus-expressing shRNA targeted to mouse OSCP was packaged with lentivirus shRNA construct (clone TRCN0000076166: 5'-CCGGGCTTCCTGAGTCCAAACCAAACTCGAGTTTGG TTTGGACTCAGGAAGCTTTTTG-3', Sigma-Aldrich), packaging vector psPAX2 (Addgene) and envelope vector pMD2.G (Addgene). Lentivirus-expressing nontarget shRNA control (SHC002, Sigma-Aldrich) was used as a control. Mouse primary neurons were cultured for 3 days before infection with lentivirus at a multiplicity of infection (m.o.i.). of 5. The virus containing medium was removed after $2 \mathrm{~h}$ and fresh culture medium was replaced to continue culturing. Neurons were treated and collected for experiments after a further 7 days in culture.

$\beta$ Subunit knockdown in mouse primary neurons. Lentivirus-expressing shRNA targeted to mouse $\beta$ subunit was packaged with lentivirus shRNA construct (clone TRCN0000076228: 5'-CCGGCTGCAACTGATCTCTCCATATCTCGAGATATG GAGAGATCAGTTGCAGTTTTTG-3', Sigma-Aldrich), packaging vector psPAX2 (Addgene) and envelope vector pMD2.G (Addgene). Lentivirus-expressing nontarget shRNA control (SHC002, Sigma-Aldrich) was used as a control. Mouse primary neurons were cultured for 3 days before infection with lentivirus at an m.o.i. of 5. The virus containing medium was removed after $2 \mathrm{~h}$ and fresh culture medium was replaced to continue culturing. Neurons were treated and collected for experiments after a further 7 days in culture.

OSCP overexpression in neurons. Human OSCP cDNA (Gene Name: ATP5O. NCBI Gene ID: 539) were inserted in to lentivirus vector with human polyubiquitin promoter-C (Addgene). Lentiviruses were packaged and applied on primary neurons similar as shRNA lentiviral vector. Oligomeric $\mathrm{A} \beta(500 \mathrm{nM})$ was used on neurons for $24 \mathrm{~h}$ before cell collection.

及 Subunit overexpression in neurons. Human $\beta$-subunit cDNA (Gene Name: $A T P 5 B$. NCBI Gene ID: 506) were inserted in to lentivirus vector with human polyubiquitin promoter-C (Addgene). Lentiviruses were packaged and applied on primary neurons similar as shRNA lentiviral vector. Oligomeric A $\beta(500 \mathrm{nM})$ was used on neurons for $24 \mathrm{~h}$ before cell collection.

Preparation and treatment of OSCP OE human neurons. Neural stem cells were plated on poly-ornithine (Sigma-Aldrich) and laminin (Life Technologies) coated culture plates in complete StemPro neural stem cell SFM (Life Technologies) at $2.5 \times 10^{4}$ cells per $\mathrm{cm}^{2}$. After $24 \mathrm{~h}$, the medium was replaced with neural differentiation medium (neurobasal A medium containing 2\% B27 and $0.5 \mathrm{mM}$ L-glutamine; Life Technologies) for neuron differentiation. 3 days after differentiation, $10 \mu \mathrm{M}$ fluorodeoxyuridine (Sigma-Aldrich) and $10 \mu \mathrm{M}$ uridine (Sigma-Aldrich) were added to remove mitotic cells. After 2 days differentiation cells were infected with expressing lentivirus at an m.o.i. of 5 , then were treated with $500 \mathrm{nM} \mathrm{A} \beta$ for 4 days after 7 days differentiation.

Mitochondrial membrane potential assay. Neurons were incubated with $200 \mathrm{nM}$ TMRM (Sigma-Aldrich) which is cell-permeable red colour fluorescent dye and a specific indicator of mitochondrial membrane potential. After the incubation of TMRM for $30 \mathrm{~min}$ in an incubator, the dye was washed by using pre-warmed neurobasal A medium and the TMRM staining on neuronal mitochondria was imaged on an inverted fluorescent microscope with on-stage incubator (Nikon). The mitochondrial membrane potential in neurites was analysed by using Nikon NIS Advanced Research software.

Mitochondrial superoxide assay. Mitochondrial superoxide was determined by using Mitosox Red (Life Technologies) ${ }^{5,26,48}$. Neurons were incubated with $2 \mu \mathrm{M}$ MitoSox Red for $30 \mathrm{~min}$ in an incubator followed by washing using pre-warmed Neurobasal A medium. The images of Mitosox Red staining were collected on a Nikon inverted confocal microscope with on-stage incubator. The intensity was subsequently analysed by using Nikon NIS Advanced Research software.

Calcein AM-cobalt chloride quenching assay. Neurons were subjected to the labelling of $1 \mu \mathrm{M}$ calcein (Life Technologies) and then incubated with $1 \mathrm{mM}$ cobalt chloride (Sigma-Aldrich) to remove cytosolic calcein staining. The changes of mitochondrial calcein were recorded in the absence or presence of ionophore, A23187 (Sigma-Aldrich) which induces mitochondrial calcium overloading on a Nikon inverted fluorescent microscope with on-stage incubator $\left(37^{\circ} \mathrm{C}, 5 \% \mathrm{CO}_{2}\right)$ for $30 \mathrm{~min}$. The results were subsequently analysed by using Nikon NIS Advanced Research software.

Neuritic mitochondrial population measurement. Primary neurons were infected by lentivirus-expressing mitochondrial targeted DsRed at 2 days in vitro (DIV). At 9-10 DIV the neurons were fixed and stained with anti-MAP2 
(Sigma-Aldrich, \#M4403, 1:400) followed by goat anti-mouse IgG conjugated with Alexa 488 (Invitrogen, \#A11029, 1:500). Images were collected under a Nikon confocal microscope. Neuritic segments $20 \mu \mathrm{m}$ away from soma were used for the analysis. Neuritic mitochondrial population was calculated as the area of a neurite occupied with mitochondria.

BN-PAGE. BN-PAGE was performed to separate mitochondrial F1FO-ATP synthase. Purified mitochondria were pelleted and resuspended in Solubilization Buffer (NativePAGE Sample Buffer (Life Technologies), Protease Inhibitor (Calbiochem, set V, EDTA free), and $1 \mathrm{mM}$ PMSF with 3.33\% Digitonin) and incubated on ice for $30 \mathrm{~min}$. Samples were centrifuged at $12,000 \mathrm{~g}$ for $10 \mathrm{~min}$ at $4{ }^{\circ} \mathrm{C}$, the supernatants was recovered and mixed with G-250 Solution $\left(\frac{1}{4}\right.$ of detergent percentage). Samples were loaded on 3-12\% NativePAGE Novex gel (Life Technologies) to separate the proteins. The intensity of coomassie staining was measured to determine the equal loading amount of samples. BN-PAGE was then transferred onto PVDF membrane with Mini-PROTEAN Tetra electrophoresis system (Bio-Rad). Proteins were subsequently fixed with 5\% acetic acid and Native Mark ladder (Life Technologies) was visualized by Ponceau S staining. Blocking was performed for $1 \mathrm{~h}$ at RT with 5\% nonfat milk. Immunoblot was performed against ATP synthase $\beta$ subunit (Santa Cruz, \#sc-33618, 1:5,000) or F1 (Abcam, \#ab109867, 1:2,000) and OSCP (Santa Cruz, \#sc-365162, 1:6,000). Images were collected on a Bio-Rad Chemidoc Imaging System. Image J software (National Institutes of Health) was used to analyse the scanned blots and to quantify protein signal intensity. For $A \beta$ treatment experiment, purified brain mitochondria were exposed to vehicle or $5 \mu \mathrm{M}$ oligomeric $\mathrm{A} \beta$ in for $30 \mathrm{~min}$ on ice and subsequently exposed to the incubation in the presence or absence of $\mathrm{Ca}^{2+}$ for $10 \mathrm{~min}$. Mitochondria were then washed with mitochondria isolation buffer via centrifugation at $8,000 \mathrm{~g}$ for $10 \mathrm{~min}$. The pelleted mitochondria were used for BN-PAGE as described above.

Dot blot. Dot blot was performed to confirm 5xFAD genotype. Mitochondrial or brain cortical extracts were loaded onto nitrocellulose membrane and allowed to dry. Membrane was blocked for $1 \mathrm{~h}$ at RT with 5\% nonfat milk. Immunoblot was performed with anti-amyloid beta IgG (CST, \#8243, 1:5,000). Images were taken with ChemiDoc MP System (Bio-Rad).

Synaptic density measurement. Synaptic density of cultured neurons was measured by counting PSD95 and vGlut1-labelled clusters attaching to neuronal dendrites and presented as the number of synapses per micron of dendrite as previously described ${ }^{67}$. Briefly, after fixation in $4 \%$ paraformaldehyde for $30 \mathrm{~min}$, neurons were blocked in $5 \%$ goat serum for $30 \mathrm{~min}$ at room temperature. PSD95, vGlut1 and MAP2 were labelled by rabbit anti-PSD95 (Cell Signaling, \#3450, 1:200), guinea pig anti-vGlut1 (Synaptic System, \#135304, 1:400) and mouse anti-MAP2 (Sigma-Aldrich, M4403, 1:400) followed by goat anti-rabbit IgG conjugated with Alexa 594 (Invitrogen, A11037, 1:500), goat anti-guinea pig IgG conjugated with Alexa 647 (Invitrogen, A21450, 1:500) and goat anti-mouse IgG conjugated with Alexa 488 (Invitrogen, A11029, 1:500), respectively. Images were collected under a Nikon confocal microscope followed by three-dimensional reconstruction by using Nikon-Elements advanced Research software ${ }^{68}$. The synapses were defined by colocalization of vGlut1 and PSD95. Dendritic segments between 20 and $50 \mu \mathrm{m}$ from the soma were used for the analysis ${ }^{69}$.

Electrophysiology. Whole-cell voltage-clamp recordings were obtained from cultured cells at room temperature using recording artificial cerebrospinal fluid (ACSF) containing (in $\mathrm{mM}$ ): $126 \mathrm{NaCl}, 2.5 \mathrm{KCl}, 1.2 \mathrm{Na}_{2} \mathrm{HPO}_{4}, 25 \mathrm{Na}_{2} \mathrm{HCO}_{3}$, 10 glucose, $2 \mathrm{CaCl}_{2}$, and $1 \mathrm{MgCl}_{2}$, bubbled with $95 \% \mathrm{O}_{2}-5 \% \mathrm{CO}_{2}$ (refs 26,70). Miniature excitatory postsynaptic currents (mEPSCs) were pharmacologically isolated by adding $75 \mu \mathrm{M}$ picrotoxin and $1 \mu \mathrm{M}$ tetrodoxin to the recording ACSF. Electrodes (2-3 M $\Omega$ open tip resistance) were filled with (in $\mathrm{mM}$ ): $130 \mathrm{CsCl}, 20$ TEA, 10 HEPES, $2 \mathrm{MgCl}_{2}$, 0.5 EGTA, 4 Na-ATP, $0.3 \mathrm{Na}$-GTP, 14 phospocreatine, and $2 \mathrm{QX}-314, \mathrm{pH} 7.2(\mathrm{CsOH})$. Recordings were performed using an Axon Multiclamp 700B amplifier (Molecular Devices, Union City, CA, USA) and the data were acquired using Axograph X (Axograph Scientific, New South Wales, Australia). Cells were recorded at a holding potential of $-65 \mathrm{mV}$, and access resistance of the recorded cells was monitored throughout the experiment and a $<20 \%$ change was deemed acceptable. The frequency and amplitude of miniature postsynaptic currents were measured from 3 min continuous recording using MiniAnalysis (Synaptosoft, Decatur, GA, USA), with a threshold set at two times the RMS baseline noise. To directly measure changes in postsynaptic glutamate receptor function, we 'puffed' glutamate onto cultured hippocampal neurons during voltage-clamp recordings. Glass pipettes (1-2 $\mu \mathrm{m}$ tip) were filled with ACSF containing $1 \mathrm{mM}$ L-glutamic acid and $0.5 \mathrm{mM}$ Alexa Fluor 594 hydrazide. Pipettes were placed within $30 \mu \mathrm{m}$ of the soma of the recorded neuron and glutamate was pressure ejected through a pneumatic drug ejection system (NPI Instruments, Tamm, F.R.G.), triggered by the recording software. Air pressure was set to $0.15-0.2 \mathrm{MPa}$ and puff duration was between 15 and $20 \mathrm{~ms}$. Fluorescence video imaging of Alexa 594 was used to ensure that glutamate puff size was comparable and that no leakage occurred between puffs. Peak amplitude was calculated from the average of at least 10 sweeps.
Statistical analysis. Two-way ANOVA followed by Bonferroni post hoc analysis or Student $t$-tests wherever appropriate were used for repeated measure analysis on SPSS software (IBM software). The distribution and variance were normal and similar in all groups. $P<0.05$ was considered significant. All data were expressed as the mean \pm s.e.m.

\section{References}

1. Reddy, P. H. Role of mitochondria in neurodegenerative diseases: mitochondria as a therapeutic target in Alzheimer's disease. CNS. Spectr. 14, 8-13 (2009).

2. Lin, M. T. \& Beal, M. F. Mitochondrial dysfunction and oxidative stress in neurodegenerative diseases. Nature 443, 787-795 (2006).

3. Swerdlow, R. H. et al. Mitochondrial dysfunction in cybrid lines expressing mitochondrial genes from patients with progressive supranuclear palsy. J. Neurochem. 75, 1681-1684 (2000).

4. Yao, J. et al. Mitochondrial bioenergetic deficit precedes Alzheimer's pathology in female mouse model of Alzheimer's disease. Proc. Natl. Acad. Sci. USA 106, 14670-14675 (2009).

5. Du, H. et al. Cyclophilin D deficiency attenuates mitochondrial and neuronal perturbation and ameliorates learning and memory in Alzheimer's disease. Nat. Med. 14, 1097-1105 (2008).

6. Lu, L. et al. Transient cerebral ischemia promotes brain mitochondrial dysfunction and exacerbates cognitive impairments in young $5 \mathrm{xFAD}$ mice. PLoS ONE 10, e0144068 (2015).

7. Trushina, E. \& McMurray, C. T. Oxidative stress and mitochondrial dysfunction in neurodegenerative diseases. Neuroscience 145, 1233-1248 (2007).

8. Cardoso, S. M., Proenca, M. T., Santos, S., Santana, I. \& Oliveira, C. R. Cytochrome c oxidase is decreased in Alzheimer's disease platelets. Neurobiol. Aging 25, 105-110 (2004).

9. Maurer, I., Zierz, S. \& Moller, H. J. A selective defect of cytochrome c oxidase is present in brain of Alzheimer disease patients. Neurobiol. Aging 21, 455-462 (2000).

10. Mutisya, E. M., Bowling, A. C. \& Beal, M. F. Cortical cytochrome oxidase activity is reduced in Alzheimer's disease. J. Neurochem. 63, 2179-2184 (1994).

11. Bosetti, F. et al. Cytochrome c oxidase and mitochondrial F1F0-ATPase (ATP synthase) activities in platelets and brain from patients with Alzheimer's disease. Neurobiol. Aging 23, 371-376 (2002).

12. Cottrell, D. A., Borthwick, G. M., Johnson, M. A., Ince, P. G. \& Turnbull, D. M. The role of cytochrome $c$ oxidase deficient hippocampal neurones in Alzheimer's disease. Neuropathol. Appl. Neurobiol. 28, 390-396 (2002).

13. Fukui, H., Diaz, F., Garcia, S. \& Moraes, C. T. Cytochrome c oxidase deficiency in neurons decreases both oxidative stress and amyloid formation in a mouse model of Alzheimer's disease. Proc. Natl Acad. Sci. USA 104, 14163-14168 (2007).

14. Liang, W. S. et al. Alzheimer's disease is associated with reduced expression of energy metabolism genes in posterior cingulate neurons. Proc. Natl Acad. Sci. USA 105, 4441-4446 (2008).

15. Terni, B., Boada, J., Portero-Otin, M., Pamplona, R. \& Ferrer, I. Mitochondrial ATP-synthase in the entorhinal cortex is a target of oxidative stress at stages I/II of Alzheimer's disease pathology. Brain Pathol. 20, 222-233 (2010).

16. Chandrasekaran, K., Hatanpaa, K., Rapoport, S. I. \& Brady, D. R. Decreased expression of nuclear and mitochondrial DNA-encoded genes of oxidative phosphorylation in association neocortex in Alzheimer disease. Brain Res. Mol. Brain Res. 44, 99-104 (1997).

17. Ding, B. et al. Gene expression profiles of entorhinal cortex in Alzheimer's disease. Am. J. Alzheimers Dis. Other Demen. 29, 526-532 (2014).

18. Wang, H. \& Oster, G. Energy transduction in the F1 motor of ATP synthase. Nature 396, 279-282 (1998)

19. Buchert, F., Schober, Y., Rompp, A., Richter, M. L. \& Forreiter, C. Reactive oxygen species affect ATP hydrolysis by targeting a highly conserved amino acid cluster in the thylakoid ATP synthase gamma subunit. Biochim. Biophys. Acta 1817, 2038-2048 (2012).

20. Dmitriev, O. Y., Jones, P. C. \& Fillingame, R. H. Structure of the subunit c oligomer in the F1Fo ATP synthase: model derived from solution structure of the monomer and cross-linking in the native enzyme. Proc. Natl Acad. Sci. USA 96, 7785-7790 (1999).

21. Alavian, K. N. et al. Bcl-xL regulates metabolic efficiency of neurons through interaction with the mitochondrial F1FO ATP synthase. Nat. Cell. Biol. 13, 1224-1233 (2011).

22. Daum, B., Walter, A., Horst, A., Osiewacz, H. D. \& Kuhlbrandt, W. Age-dependent dissociation of ATP synthase dimers and loss of inner-membrane cristae in mitochondria. Proc. Natl Acad. Sci. USA 110, 15301-15306 (2013).

23. Alavian, K. N. et al. An uncoupling channel within the c-subunit ring of the F1FO ATP synthase is the mitochondrial permeability transition pore. Proc. Natl Acad. Sci. USA 111, 10580-10585 (2014).

24. Halestrap, A. P. The $C$ ring of the F1Fo ATP synthase forms the mitochondrial permeability transition pore: a critical appraisal. Front. Oncol. 4, 234 (2014). 
25. Baines, C. P. et al. Loss of cyclophilin D reveals a critical role for mitochondrial permeability transition in cell death. Nature 434, 658-662 (2005).

26. Du, H. et al. Cyclophilin D deficiency rescues Abeta-impaired PKA/CREB signaling and alleviates synaptic degeneration. Biochim. Biophys. Acta 1842, 2517-2527 (2014).

27. Oakley, H. et al. Intraneuronal beta-amyloid aggregates, neurodegeneration, and neuron loss in transgenic mice with five familial Alzheimer's disease mutations: potential factors in amyloid plaque formation. J. Neurosci. 26, 10129-10140 (2006)

28. Gillessen, T., Grasshoff, C. \& Szinicz, L. Mitochondrial permeability transition can be directly monitored in living neurons. Biomed. Pharmacother. 56, 186-193 (2002)

29. Przygodzki, T., Sokal, A. \& Bryszewska, M. Calcium ionophore A23187 action on cardiac myocytes is accompanied by enhanced production of reactive oxygen species. Biochim. Biophys. Acta 1740, 481-488 (2005).

30. Dupuis, A., Lunardi, J., Issartel, J. P. \& Vignais, P. V. Interactions between the oligomycin sensitivity conferring protein (OSCP) and beef heart mitochondrial F1-ATPase. 2. Identification of the interacting F1 subunits by cross-linking. Biochemistry 24, 734-739 (1985).

31. Giorgio, V. et al. Cyclophilin D modulates mitochondrial F0F1-ATP synthase by interacting with the lateral stalk of the complex. J. Biol. Chem. 284, 33982-33988 (2009).

32. Giorgio, V. et al. Dimers of mitochondrial ATP synthase form the permeability transition pore. Proc. Natl Acad. Sci. USA 110, 5887-5892 (2013).

33. Du, H. et al. Early deficits in synaptic mitochondria in an Alzheimer's disease mouse model. Proc. Natl Acad. Sci. USA 107, 18670-18675 (2010).

34. Ardiles, A. O. et al. Postsynaptic dysfunction is associated with spatial and object recognition memory loss in a natural model of Alzheimer's disease. Proc. Natl Acad. Sci. USA 109, 13835-13840 (2012).

35. Lustbader, J. W. et al. ABAD directly links Abeta to mitochondrial toxicity in Alzheimer's disease. Science 304, 448-452 (2004).

36. Moreira, P. I., Santos, M. S., Moreno, A. \& Oliveira, C. Amyloid beta-peptide promotes permeability transition pore in brain mitochondria. Biosci. Rep. 21, 789-800 (2001)

37. Ferreira, S. T. \& Klein, W. L. The Abeta oligomer hypothesis for synapse failure and memory loss in Alzheimer's disease. Neurobiol. Learn. Mem. 96, 529-543 (2011).

38. Buskila, Y., Crowe, S. E. \& Ellis-Davies, G. C. Synaptic deficits in layer 5 neurons precede overt structural decay in 5xFAD mice. Neuroscience 254, 152-159 (2013)

39. Nieweg, K., Andreyeva, A., van Stegen, B., Tanriover, G. \& Gottmann, K. Alzheimer's disease-related amyloid-beta induces synaptotoxicity in human iPS cell-derived neurons. Cell Death Dis. 6, e1709 (2015).

40. Manczak, M., Calkins, M. J. \& Reddy, P. H. Impaired mitochondrial dynamics and abnormal interaction of amyloid beta with mitochondrial protein Drp1 in neurons from patients with Alzheimer's disease: implications for neuronal damage. Hum. Mol. Genet. 20, 2495-2509 (2011).

41. Devenish, R. J., Prescott, M., Boyle, G. M. \& Nagley, P. The oligomycin axis of mitochondrial ATP synthase: OSCP and the proton channel. J. Bioenerg. Biomembr. 32, 507-515 (2000).

42. Kane, L. A., Youngman, M. J., Jensen, R. E. \& Van Eyk, J. E. Phosphorylation of the $\mathrm{F}(1) \mathrm{F}(\mathrm{o})$ ATP synthase beta subunit: functional and structural consequences assessed in a model system. Circ. Res. 106, 504-513 (2010).

43. Lemaire, C. \& Dujardin, G. Preparation of respiratory chain complexes from Saccharomyces cerevisiae wild-type and mutant mitochondria: activity measurement and subunit composition analysis. Methods Mol. Biol. 432, 65-81 (2008).

44. Joshi, S., Cao, G. J., Nath, C. \& Shah, J. Oligomycin sensitivity conferring protein (OSCP) of bovine heart mitochondrial ATP synthase: high-affinity OSCP-Fo interactions require a local alpha-helix at the C-terminal end of the subunit. Biochemistry 36, 10936-10943 (1997).

45. Antoniel, M. et al. The oligomycin-sensitivity conferring protein of mitochondrial ATP synthase: emerging new roles in mitochondrial pathophysiology. Int. J. Mol. Sci. 15, 7513-7536 (2014).

46. Ly, C. V. \& Verstreken, P. Mitochondria at the synapse. Neuroscientist 12, 291-299 (2006).

47. Selkoe, D. J. Soluble oligomers of the amyloid beta-protein impair synaptic plasticity and behavior. Behav. Brain. Res. 192, 106-113 (2008).

48. Guo, L. et al. Cyclophilin D deficiency rescues axonal mitochondrial transport in Alzheimer's neurons. PLoS ONE 8, e54914 (2013).

49. Smith, S. M. et al. Calcium regulation of spontaneous and asynchronous neurotransmitter release. Cell Calcium 52, 226-233 (2012).

50. Hansson Petersen, C. A. et al. The amyloid beta-peptide is imported into mitochondria via the TOM import machinery and localized to mitochondrial cristae. Proc. Natl Acad. Sci. USA 105, 13145-13150 (2008).

51. Roher, A. E. et al. Amyloid beta peptides in human plasma and tissues and their significance for Alzheimer's disease. Alzheimer's Dement. 5, 18-29 (2009).
52. Li, Q. X. et al. Secretion of Alzheimer's disease Abeta amyloid peptide by activated human platelets. Lab. Invest. 78, 461-469 (1998).

53. Swerdlow, R. H., Burns, J. M. \& Khan, S. M. The Alzheimer's disease mitochondrial cascade hypothesis: progress and perspectives. Biochim. Biophys. Acta 1842, 1219-1231 (2014).

54. Joachim, C. L., Mori, H. \& Selkoe, D. J. Amyloid beta-protein deposition in tissues other than brain in Alzheimer's disease. Nature 341, 226-230 (1989).

55. Mutisya, E. M., Bowling, A. C. \& Beal, M. F. Cortical cytochrome oxidase activity is reduced in Alzheimer's disease. J. Neurochem. 63, 2179-2184 (1994).

56. Martinez-Cano, E. et al. [Functional disorders of FOF1-ATPase in submitochondrial particles obtained from platelets of patients with a diagnosis of probable Alzheimer's disease]. Rev. Neurol. 40, 81-85 (2005).

57. Elrod, J. W. et al. Cyclophilin D controls mitochondrial pore-dependent $\mathrm{Ca}(2+)$ exchange, metabolic flexibility, and propensity for heart failure in mice. J. Clin. Invest. 120, 3680-3687 (2010).

58. Ichas, F. \& Mazat, J. P. From calcium signaling to cell death: two conformations for the mitochondrial permeability transition pore. Switching from low- to high-conductance state. Biochim. Biophys. Acta 1366, 33-50 (1998).

59. Spat, A., Szanda, G., Csordas, G. \& Hajnoczky, G. High- and low-calciumdependent mechanisms of mitochondrial calcium signalling. Cell Calcium 44, 51-63 (2008).

60. Schmidt, C. et al. Amyloid precursor protein and amyloid beta-peptide bind to ATP synthase and regulate its activity at the surface of neural cells. Mol. Psychiatry 13, 953-969 (2008).

61. Cha, M. Y. et al. Mitochondrial ATP synthase activity is impaired by suppressed O-GlcNAcylation in Alzheimer's disease. Hum. Mol. Genet. 24, 6492-6504 (2015).

62. Pullman, M. E., Penefsky, H. S., Datta, A. \& Racker, E. Partial resolution of the enzymes catalyzing oxidative phosphorylation I. Purification and properties of soluble, dinitrophenol-stimulated adenosine triphosphatase. J. Biol. Chem. 235, 3322-3329 (1960).

63. De Vries, S. J., van Dijk, M. \& Bonvin, A. M. The HADDOCK web server for data-driven biomolecular docking. Nat. Protoc. 5, 883-897 (2010).

64. DeLano, W. L. The PyMOL molecular graphics system (DeLano Scientific, 2002).

65. Stelzer, A. C. et al. NMR studies of an immunomodulatory benzodiazepine binding to its molecular target on the mitochondrial F1F0-ATPase. Biopolymers 93, 85-92 (2010).

66. Giorgio, V. et al. Dimers of mitochondrial ATP synthase form the permeability transition pore. Proc. Natl Acad. Sci. USA 110, 5887-5892 (2013).

67. Li, H., Zhong, X., Chau, K. F., Williams, E. C. \& Chang, Q. Loss of activity-induced phosphorylation of MeCP2 enhances synaptogenesis, LTP and spatial memory. Nat. Neurosci. 14, 1001-1008 (2011).

68. Wang, L. et al. Synaptosomal mitochondrial dysfunction in 5xFAD mouse model of Alzheimer's disease. PLoS ONE 11, e0150441 (2016).

69. de Jong, A. P., Schmitz, S. K., Toonen, R. F. \& Verhage, M. Dendritic position is a major determinant of presynaptic strength. J. Cell. Biol. 197, 327-337 (2012).

70. Kroener, S., Chandler, L. J., Phillips, P. E. \& Seamans, J. K. Dopamine modulates persistent synaptic activity and enhances the signal-to-noise ratio in the prefrontal cortex. PLoS ONE 4, e6507 (2009).

\section{Acknowledgements}

We thank Dr Mark Richter for advices on the experiments on F1FO-ATP synthase and the critical reading of the manuscript. We thank Dr Gail Breen for the insightful discussion. We thank Ms. Janani Ramachandra and Brittney Campbell for mouse genotyping. We thank the UT Southwestern Medical Center ADC Neuropathology Core supported by ADC grant (AG12300) to provide human brain tissues. This study is supported by research funding from NIH (R00AG037716), NSFC (31271145, 81200847) and SDNSF (JQ201318). LG was supported by Alzheimer's Association (NIRG-12-242803). JMP was supported by NS077015.

\section{Author contributions}

H.D. and L.G. initiated the study. H.D., L.G., S.K. and J.M.P. designed the study. S.B., L.G., A.P., J.T., L.W., N.T., E.G., L.L., S.K. and H.D. performed experiments. S.B., L.G., A.P., S.K. and H.D. analysed the data. H.D. prepared the manuscript.

\section{Additional information}

Supplementary Information accompanies this paper at http://www.nature.com/ naturecommunications

Competing financial interests: The authors declare no competing financial interests. 
Reprints and permission information is available online at http://npg.nature.com/ reprintsandpermissions/

How to cite this article: Beck, S. J. et al. Deregulation of mitochondrial F1FO-ATP synthase via OSCP in Alzheimer's disease. Nat. Commun. 7:11483 doi: $10.1038 /$ ncomms11483 (2016). (c) (i) This work is licensed under a Creative Commons Attribution 4.0 International License. The images or other third party material in this article are included in the article's Creative Commons license, unless indicated otherwise in the credit line; if the material is not included under the Creative Commons license, users will need to obtain permission from the license holder to reproduce the material. To view a copy of this license, visit http://creativecommons.org/licenses/by/4.0/ 